\title{
Actin binding domain of Rng2 sparsely bound on F-actin strongly inhibits actin movement on myosin II
}

Running title: Rng2CHD inhibits acto-myosin II movement

Yuuki Hayakawa ${ }^{1,6}$, Masak Takaine ${ }^{2,6, \#, \text { Taiga Imai }^{3}, \text { Masafumi D. Yamada }^{4} \text {, Keiko Hirose }}{ }^{2,4}$,

Kien Xuan Ngo ${ }^{5}$, Noriyuki Kodera ${ }^{5}$, Kiyotaka Tokuraku ${ }^{3}$, Osamu Numata ${ }^{2}$, Kentaro

Nakano $^{2, *}$, Taro Q.P. Uyeda ${ }^{1,2,4, *}$

${ }^{1}$ Department of Physics, Faculty of Science and Engineering, Graduate School of Waseda

University, Shinjuku, Tokyo 169-8555, Japan

${ }^{2}$ Department of Biology, Degree Programs in Life and Earth Sciences, Graduate School of Science

and Technology, University of Tsukuba, Tsukuba, Ibaraki 305-8572, Japan

${ }^{3}$ Department of Applied Sciences, Muroran Institute of Technology, Muroran, Hokkaido 050-8585,

Japan

${ }^{4}$ Biomedical Research Institute, National Institute of Advanced Industrial Science and Technology,

Tsukuba, Ibaraki 305-8565, Japan

${ }^{5}$ WPI Nano Life Science Institute, Kanazawa University, Kanazawa, Ishikawa 920-1192, Japan,

${ }^{6}$ These authors contributed equally.

\#Present addresses: Gunma University Initiative for Advanced Research (GIAR) and Institute for

Molecular and Cellular Regulation (IMCR), Gunma University, Maebashi 371-8512, Japan 
bioRxiv preprint doi: https://doi.org/10.1101/2020.04.14.041046; this version posted May 23, 2020. The copyright holder for this preprint (which was not certified by peer review) is the author/funder, who has granted bioRxiv a license to display the preprint in perpetuity. It is made available under aCC-BY-NC 4.0 International license.

*Correspondence: t-uyeda@waseda.jp and knakano@biol.tsukuba.ac.jp 


\section{Abstract}

The contraction of contractile rings (CRs) depends on interaction between actin filaments and myosin II filaments. The rate of contraction in the fission yeast Schizosaccharomyces pombe is less than $1 / 120$ of the velocity of acto-myosin II movement in vitro, but the mechanism of inhibition has not been described. Here, we found that the calponin-homology actin binding domain of fission yeast IQGAP Rng2 (Rng2CHD) strongly inhibits the motility of actin filaments on skeletal muscle myosin II fragments in vitro, even at a low ratio of bound Rng2CHD to actin protomers, reducing the sliding velocity to half when the binding ratio was $1 / 75$. Rng2CHD also induced structural changes of actin filaments and reduced the affinity between actin filaments and subfragment 1 (S1) of muscle myosin II carrying ADP. Intriguingly, actin-activated ATPase of S1 was only mildly inhibited, even by high concentrations of Rng2CHD. Moreover, the motility of actin filaments by myosin V was not inhibited by Rng2CHD. We propose a new regulatory mechanism for acto-myosin II movement that involves Rng2CHD-induced structural changes of actin filaments. 


\section{Introduction}

Actin exists in all eukaryotic cells and performs a wide variety of functions. One of these

functions is the contraction of contractile rings (CRs). The CR is a ring-like structure that appears

transiently on the equatorial plane underneath the cell membrane during cytokinesis of animal cells

and many unicellular eukaryotes. CR mainly consists of actin filaments and myosin II filaments. It is

thought that a CR contracts by sliding between the two filament systems due to actomyosin movement

$(1,2)$. However, how contraction is regulated remains ambiguous. For example, the circumferential

contraction rate of the CR of the fission yeast Schizosaccharomyces pombe, a model organism for

cytokinesis studies, is $0.5 \mu \mathrm{m} / \mathrm{min}$ (3), while actin filaments on myosin II derived from $S$. pombe move

at a rate of $30 \mu \mathrm{m} / \mathrm{min}$ in vitro (4). This 60 -fold difference in speed is most likely an underestimate of

the extent of inhibition, as explained next. CRs have actin filaments of opposite polarities, as is

expected for contraction in a manner similar to muscle sarcomeres or stress fibers, but they are not as

regularly organized as in sarcomeres or stress fibers (5). Nonetheless, to simplify the argument, if we

assume that CRs consist of contraction units, each of which contains myosin II filaments and anti-

parallel actin filaments, and there are $\mathrm{N}$ of those contractile units in series in a $\mathrm{CR}$, then the maximum

contraction rate would be the sliding velocity in vitro multiplied by a factor of $2 \mathrm{~N}$. Therefore, it is

estimated that the contraction rate of the CR in $S$. pombe is inhibited to less than $0.5 /(30 \times 2)=1 / 120$ of

the velocity of acto-myosin II movement in vitro, even when assuming the extreme case of $\mathrm{N}=1$. In 
the cellular slime mold Dictyostelium discoideum, the contractile velocity of the CR is $0.18 \mu \mathrm{m} / \mathrm{s}(6)$,

whereas the sliding velocity of actin filaments on myosin II derived from D. discoideum is $1.4 \mu \mathrm{m} / \mathrm{s}$

in vitro (7). These results suggest that contraction of the CR is regulated in an inhibitory manner in

cells with or without a cell wall. Two distinct mechanisms may contribute to this significant inhibition.

First, the increase in membrane surface tension due to membrane deformation and high viscoelasticity

of the cytoplasm may create high loads that slow down the movement speed of myosin II, as was

demonstrated for skeletal muscle contraction (8). The other possibility is that myosin II motor activity

is negatively regulated in CRs. In this study, we experimentally examined the latter hypothesis. Several

actin-binding proteins (ABPs), such as formin, tropomyosin and Rng2, play important roles in the

formation of CRs in S. pombe (9), and one of those ABPs may be involved in the inhibitory regulation

of the contraction rate of CRs. For example, overexpession of $\alpha$-actinin in mammalian cells

significantly slows cytokinesis (10). However, the contraction rate of CRs was not affected by

knockout or overexpression of $\alpha$-actinin in $S$. pombe (11). This suggested that, in $S$. pombe, an ABP

other than $\alpha$-actinin slows contraction of CRs, either singly or in a functionally redundant manner with

$\alpha$-actinin. Rng2, an IQ motif-containing GTPase activating protein (IQGAP), which has a 181 amino

acid residue-long calponin-homology actin binding domain (CHD) at the N-terminus $(9,12)$, is a

prime candidate for this regulator. It is thought that Rng2 crosslinks and bundles actin filaments

together to form CRs, because CRs were not formed in Rng2 knockout S. pombe cells even though 
actin filaments were assembled at their division sites $(9,12)$. Additionally, temperature-sensitive Rng2 mutant cells showed normal actin filament rings at the permissive temperature but the distribution of myosin II on actin filaments was abnormal (12). This result suggested that Rng2 regulates the interaction between actin filaments and myosin II filaments. In this study, therefore, we investigated the possible role of CHD in Rng2 (Rng2CHD) as the regulator of acto-myosin movement.

For this purpose, we performed in vitro motility assays in which actin filaments move on myosin II HMM to examine if Rng2CHD inhibits acto-myosin motility. We found that Rng2CHD bound on actin filaments strongly inhibited acto-myosin II motility even when binding was sparse, without significantly inhibiting ATPase activity. Furthermore, the affinity of actin filaments sparsely decorated by Rng2CHD displayed a reduced affinity for the motor domain of myosin II carrying ADP, demonstrating that this is a novel mode of regulation of acto-myosin II motility. 


\section{Results}

\section{Rng2CHD strongly inhibits sliding of actin filaments on myosin II HMM but not on myosin V}

\section{НMМ in vitro}

To examine if Rng2 inhibits actomyosin motility, we performed in vitro motility assays in which actin filaments move on a glass surface coated with heavy meromyosin (HMM) of skeletal muscle myosin II (7). The movement of actin filaments was strongly inhibited by Rng2CHD, the actinbinding domain of Rng2, and was completely stopped in the presence of $200 \mathrm{nM}$ Rng2CHD (Fig. 1A). A trajectory analysis showed that, under all the inhibition conditions tested, the front end of the actin filament was followed by the remainder of the filament (Fig. 1B). Buckling of the filaments, indicative of local inhibition of movement, was rarely observed. This indicates that movement is more or less uniformly inhibited along the entire length of the filaments.

We also performed in vitro motility assays in which actin filaments moved on recombinant myosin V HMM that was expressed in insect cells. In striking contrast with myosin II, up to $1 \mu \mathrm{M}$ Rng2CHD did not inhibit the sliding of actin filaments on myosin V HMM (Fig. 1A).

Rng2CHD on actin filaments inhibits acto-myosin II movement in vitro even when binding is sparse

We estimated the binding ratio of Rng2CHD to actin protomers when the movement of 
actin filaments on myosin II HMM was inhibited. The concentrations of Rng2CHD that caused 50\%, $75 \%$ and $95 \%$ reduction of movement speed were estimated from the velocity curve to be $12.4 \mathrm{nM}$, $31.6 \mathrm{nM}$ and $71.4 \mathrm{nM}$, respectively (Fig. 1A). In parallel, we performed co-sedimentation assays of actin filaments with Rng2CHD, and the dissociation constant $\left(K_{d}\right)$ between Rng2CHD and actin protomers was calculated (Fig. 2A, 2B). $K_{d}$ was determined to be $0.92 \mu \mathrm{M}$ by the following fitting function: $\left[{\left.\text { Rng } 2 C H D_{\text {bound }}\right]}\right]=\left[\right.$ Actin $\left._{\text {total }}\right][$ Rng2CHD free $] /[$ Rng2CHD free $\left.]+K_{d}\right) \quad($ Eq. 1 in Materials and Methods). In in vitro motility assays, where the concentration of actin protomers is much lower than that of Rng2CHD, the following approximation holds: $\left[\right.$ Rng $\left.2 C H D_{\text {bound }}\right] /\left[\right.$ Actin $\left._{\text {free }}\right] \cong$ $\left[R n g 2 C H D_{\text {total }}\right] / K_{d}$ (Eq. 3 in Materials and Methods). Using this approximation, the binding ratio of Rng2CHD to actin protomers that caused $50 \%, 75 \%$ and $95 \%$ reduction of acto-myosin II movement speed was estimated to be $1 / 75,1 / 30$ and $1 / 13$, respectively (Table 1 ). In other words, Rng2CHD was sparsely bound to actin filaments (1.3\% occupancy) when the speed was reduced to half, and $7.7 \%$ occupancy even when it inhibited $95 \%$ of movement.

To directly confirm that sparsely bound Rng2CHD potently inhibits actomyosin movements, we prepared Rng2CHD fused with GFP to its N-terminus via a 16-residue linker (GFPRng2CHD), and determined the binding ratio of GFP-Rng2CHD to actin protomers from the fluorescence intensity of GFP. GFP-Rng2CHD strongly inhibited actin filament movement on myosin II HMM in a manner similar to Rng2CHD (Fig. 3A). We then measured the fluorescence intensity of 
bound GFP-Rng2CHD per unit length of actin filaments. The fluorescence intensity increased depending on the GFP-Rng2CHD concentration in the buffer, and was saturated in the presence of 5,000 nM GFP-Rng2CHD (Fig. 3B, 3C). The co-sedimentation assay showed that one molecule of GFP-Rng2CHD binds to one molecule of an actin protomer in the presence of 5,000 nM GFPRng2CHD (Fig. 3D). Therefore, we regarded this saturated fluorescence intensity as a one-to-one binding state, and used it as the reference to calculate how much GFP-Rng2CHD binds to actin protomers when movements were inhibited. This fluorescence-based direct quantification also demonstrated that sparsely bound GFP-Rng2CHD strongly inhibits acto-myosin II movement (Table 1), although based on these directly measured binding ratios of GFP-Rng2CHD to actin protomers, higher binding ratio was needed to obtain the same degree of motility inhibition than that estimated from $K_{d}$ using unlabeled Rng2CHD.

\section{Rng2CHD locally kinks or distorts actin filaments}

Rng2CHD reduced the rate of acto-myosin II movement to $50 \%$ at a very low binding ratio,

estimated to be $1 / 75$ from $K_{d}$ (Fig. $1 \mathrm{~A}$ and Table 1 ). Thus, we inferred that sparsely bound Rng2CHD somehow induces global structural changes in actin filaments and inhibits acto-myosin II movement.

To examine whether sparsely bound Rng2CHD actually changes the structure of actin filaments, we observed actin filaments in the presence of Rng2CHD using negative stain electron microscopy. In the 
absence of Rng2CHD, double helical filaments typical of actin filaments were observed (Fig. 4A), but the helical structures became irregular and the filaments often looked locally distorted in the presence of Rng2CHD (Fig. 4B-4D). In some portions of the filaments, the two actin protofilaments appeared to be separated so that a dark straight line was observed in between (indicated by yellow brackets), suggesting that the interactions between neighboring actin protomers are altered. At a higher concentration $(1 \mu \mathrm{M})$ of Rng2CHD, the filaments were frequently kinked or even fragmented. The binding ratios of Rng2CHD to actin protomers were estimated to be $1 / 25$ and $1 / 5$ at $40 \mathrm{nM}$ and 200 nM of Rng2CHD, respectively, based on $K_{d}$ (Eq. 2 in Materials and Methods). These results indicate that when low concentrations of Rng2CHD was allowed to interact with actin filaments, structural changes are induced even in actin protomers not in direct contact with Rng2CHD molecules.

\section{Steady state actin-activated S1 ATPase is only weakly inhibited by Rng2CHD}

To gain insight into the mechanism by which structural changes of actin filaments induced

by Rng2CHD inhibits actomyosin motility, we investigated the effects of Rng2CHD on actin-activated

ATPase activity of myosin II subfragment-1 (S1). Actin-activated S1 ATPase was moderately inhibited (approximately $50 \%$ ) by the highest concentration of Rng2CHD tested (5 $\mu \mathrm{M}$; Fig. 5). In the presence of $0.33 \mu \mathrm{M}, 0.82 \mu \mathrm{M}$ and $1.91 \mu \mathrm{M}$ of Rng2CHD and $23.8 \mu \mathrm{M}$ of actin filaments, the binding ratios of Rng2CHD to actin were calculated to be $1 / 75,1 / 30$, and 1/13, respectively (Eq. 2 in Materials and 
Methods). These binding ratios caused a $50 \%, 75 \%$ and $95 \%$ reduction in the speed of actomyosin movement (Fig. 1A and Table 1). However, the inhibition of actin-activated S1 ATPase activity was much weaker and disproportional to the inhibition of movement (Fig. 5). This indicates that even when actomyosin movement was strongly inhibited or halted by Rng2CHD, the myosin II ATPase cycle was running, and was only partially inhibited.

\section{Rng2CHD inhibits the steady-state binding of S1 to actin filaments in the presence of ADP, but}

\section{not in the presence of ATP}

We examined the possibility that Rng2CHD might affect the affinity between actin filaments and myosin II when it inhibits acto-myosin II motility. First, we performed a co-sedimentation assay of actin filaments and S1, and found that Rng2CHD did not significantly inhibit steady-state binding of $\mathrm{S} 1$ to actin filaments in the presence of ATP as in in vitro motility assays (Fig. 6A, 6C). However, Rng2CHD weakly but statistically significantly inhibited the binding of S1• ADP to actin filaments in the presence of ADP in the buffer (Fig. 6B, 6C).

A co-sedimentation assay using myosin II filaments showed that Rng2CHD did not bind to myosin II under the conditions employed in the in vitro motility assays (Supplemental Fig. 1). 
We previously reported that when HMM of $D$. discoideum myosin II fused with GFP was

allowed to interact with actin filaments in the presence of a very low concentration of ATP, HMM-

GFP forms clusters along actin filaments $(13,14)$. This was interpreted to represent local polymorphism of actin filaments, such that some segments of the filaments have a higher affinity for HMM than other parts of the filament, and HMM-GFP preferentially repeats transient binding to those segments. In the presence of a high concentration of ATP, no fluorescent clusters were observed, and in the absence of ATP, HMM-GFP uniformly bound along the entire filaments (13).

We speculated that, for the detectable fluorescent clusters to form, HMM needs to bind to actin filaments repetitively and transiently but the dwell time of each binding event must be long enough to allow visualization. Based on this hypothesis, we observed how Rng2CHD affects the formation of myosin II HMM-GFP clusters along actin filaments in the presence of a very low concentration of ATP using fluorescence microscopy. Numerous fluorescent spots, each representing a HMM-GFP cluster, were observed along actin filaments in the presence of $0.5 \mu \mathrm{M}$ ATP and the absence of Rng2CHD. In the presence of $1 \mathrm{nM}$ Rng2CHD, the number and fluorescent intensity of fluorescent spots were significantly reduced, and fluorescent spots were apparently absent in the presence of $10 \mathrm{nM}$ Rng2CHD (Fig. 7A). In contrast, Rng2CHD did not affect the binding of HMMGFP to actin filaments in the nucleotide-free state (Fig. 7B). 


\section{Rng2CHD significantly decreases the number of S1 molecules transiently bound to actin}

\section{filaments in the presence of ATP}

We also directly observed transient binding of S1 molecules to actin filaments in the presence of ATP using high-speed atomic force microscopy (HS-AFM). At a scan speed of $0.5 \mathrm{~s}$ per field of view, transient binding of S1 to actin filaments was rarely observed in the presence of $500 \mu \mathrm{M}$ ATP alone, but was frequently observed in the presence of $50 \mu \mathrm{M}$ ATP and $1 \mathrm{mM}$ ADP. S1 molecules were easily identified based on their size and shape, while Rng2CHD molecules were too small to visualize under our experimental condition. We analyzed images scanned between 1 and 2 min after the addition of S1 or ATP, and visually counted the number of transiently bound S1 molecules (Fig. 8A). The binding dwell time of S1 molecules on the top of the filament was shorter than those bound along the sides of the filaments. Therefore, we separately counted the S1 molecules bound on the top and along the sides of the filaments. The number of S1 molecules that transiently bound to actin filaments was significantly decreased when $12.4 \mathrm{nM}$ Rng2CHD, the concentration that caused 50\% inhibition of motility, was added before the addition of S1 (Fig. 8B, 8C). It was thus directly confirmed that sparsely bound Rng2CHD affected the binding of S1 to actin filaments in the presence of ATP and ADP. In contrast, when S1 was strongly bound to actin filaments in the nucleotide-free state in advance, and then $50 \mu \mathrm{M}$ ATP, $1 \mathrm{mM}$ ADP and $12.4 \mathrm{nM}$ Rng2CHD were added, the number of S1 molecules transiently bound to actin filaments was unaffected by Rng2CHD (Fig. 8C). Thus, there is 
bioRxiv preprint doi: https://doi.org/10.1101/2020.04.14.041046; this version posted May 23, 2020. The copyright holder for this preprint (which was not certified by peer review) is the author/funder, who has granted bioRxiv a license to display the preprint in perpetuity. It is made available under aCC-BY-NC 4.0 International license.

apparent hysteresis in the impact of Rng2CHD on S1 binding to actin filaments, depending on whether

S1 or Rng2CHD actin filaments first encounter. 


\section{Discussion}

\section{Structural changes of actin filaments induced by sparsely bound Rng2CHD inhibit acto-myosin}

\section{II movement}

Rng2CHD, the actin-binding domain of Rng2, strongly inhibited acto-myosin II motility along the entire length of the filament, when actin filaments were estimated to be only sparsely decorated by Rng2CHD (Fig. 1A and Table 1). Furthermore, fluorescence measurements unambiguously showed that sparsely bound GFP-Rng2CHD strongly inhibited acto-myosin II movement (Fig. 3A, 3D). Since binding of Rng2CHD or GFP-Rng2CHD was sparse, the inhibition of motility could not be due to steric hindrance or direct competition for a binding site on actin molecules.

We thus inferred that sparsely bound Rng2CHD and GFP-Rng2CHD induced some structural changes in actin filaments, and these inhibited the productive interaction between actin filaments and myosin II. Previous studies advocated that structural changes of actin filaments modulate affinities for ABPs. For example, cooperative structural changes of actin filaments induced by myosin II and cofilin contribute to mutually exclusive cooperative binding of myosin II and cofilin to actin filaments (15). Meanwhile, in this study, electron microscopy observations showed that Rng2CHD significantly changed the structure of actin filaments even when Rng2CHD was only sparsely bound to actin filaments (Fig. 4). The images of actin filaments suggest that Rng2CHD somehow reduces, or at least changes, the interaction between the two protofilaments even when the binding ratio of Rng2CHD to 
actin protomers was only $1 / 25$. Thus, it was actually confirmed that Rng2CHD alters wide areas of the structure of actin filaments even when its binding ratio to actin protomers is very low. At the saturating concentration of Rng2CHD (1 $\mu \mathrm{M})$, actin filaments became kinked, distorted or fragmented (Fig. 4D), which can also be explained by altered interactions between neighboring actin protomers.

We consider two possible mechanisms how sparsely bound Rng2CHD inhibits acto-myosin II movements. The first mechanism proposes that one or two actin protomers in direct contact with the bound Rng2CHD molecule undergo structural changes, and those affected actin protomers bind persistently to myosin II motors even in the presence of ATP, acting as a potent break. The second mechanism assumes that a bound Rng2CHD molecule changes the structure of multiple actin protomers, and the affected actin protomers become unable to interact with myosin II productively. The first mechanism predicts that Rng2CHD should increase the amount of co-sedimented S1 in the presence of ATP. However, this was not the case (Fig. 6). Furthermore, the buckling of the moving actin filaments on HMM-coated surfaces, indicative of local inhibition of the movement, was rarely observed in the presence of various concentrations of Rng2CHD (Fig. 1B). In the presence of $5 \mathrm{nM}$ Rng2CHD, movement speed was inhibited by $40 \%$, and the binding ratio to actin protomers was estimated as $1 / 185$ based on $K_{d}$ (Eq. 3 in Materials and Methods). In other words, on average, one molecule of Rng2CHD is bound per $500 \mathrm{~nm}$ of actin filament. A buckling of this length would have been detectable with a spatial resolution of about $380 \mathrm{~nm}$ calculated from the numerical aperture and 
the fluorescence wavelength of this observation. Those two reasons led us to reject the first mechanism and conclude that force generation by myosin II is inhibited in broad sections of actin filaments not in direct contact with Rng2CHD. The velocity of acto-myosin II movement would decrease as the proportion of the inhibited section within the filaments increases in a Rng2CHD concentrationdependent manner. This idea is supported by the fact that the number of fluorescent spots of HMMGFP was significantly reduced in a Rng2CHD concentration-dependent manner in the presence of a very low concentration of ATP (Fig. 7A).

Hereafter, we resolve the inhibition process by two aspects, and discuss their mechanisms next. The first is the mechanism by which sparsely bound Rng2CHD causes global structural changes in actin filaments. The second aspect is the mechanism by which structural changes of actin filaments inhibit actin motility driven by myosin II.

\section{The mechanism by which Rng2CHD causes global structural changes in actin filaments}

We now consider two hypotheses for the mechanism by which one molecule of Rng2CHD changes the structure of multiple actin protomers. The first is a cooperative structural change, in which one molecule of Rng2CHD bound to one actin protomer changes the structure of multiple neighboring actin protomers in the same filament. Such cooperative propagation of conformational changes has been reported for many ABPs. For example, cofilin binds to actin filaments cooperatively to form 
clusters along the filaments, and the half helical pitch of actin filaments in the cluster is reduced by $25 \%$ (16). Notably, this structural change was propagated to the neighboring cofilin-unbound bare region $(17,18)$. Moreover, a single molecule of gelsolin bound to the barbed end of an actin filament changes the structure of all actin protomers in the filament (19). Other ABPs such as myosin II (20), tropomyosin (21), $\alpha$-actinin (22) and formin (23) also cause cooperative structural changes of actin filaments. The other hypothesis is the memory effect, in which Rng2CHD molecules repeat transient binding to different actin protomers, and the actin protomers remain in the altered conformation for a certain period of time after dissociation of Rng2CHD. If there is a memory effect, Rng2CHD can alter the structure of entire actin filaments even when its binding ratio to actin protomers is low. We speculate that either or both of the two mechanisms, cooperative structural change and memory effect, underlie the global structural changes in actin filaments induced by sparsely bound Rng2CHD.

\section{The mechanism by which structural changes of actin filaments inhibit acto-myosin II motility}

Rng2CHD inhibited steady-state binding of S1•ADP to actin filaments (Fig. 6B, 6C). In other words, Rng2CHD reduced the affinity between S1•ADP and actin filaments. We also observed transient binding of HMM or S1 in the presence of ATP using a fluorescent microscope and HS-AFM.

Fluorescent microscopy demonstrated that Rng2CHD significantly decreased the region along actin filaments where HMM-GFP fluorescence was observed in the presence of $0.5 \mu \mathrm{M}$ ATP (Fig. 7A). 
Moreover, HS-AFM directly showed that Rng2CHD significantly reduced the number of imaged S1 molecules transiently bound to actin filaments in the presence of $50 \mu \mathrm{M}$ ATP and $1 \mathrm{mM}$ ADP (Fig. 8).

First, we consider the results of high-speed AFM observation. Unlike when excess ADP coexists in the buffer, S1 molecules transiently bound to actin filaments were hardly observed in the presence of $500 \mu \mathrm{M}$ ATP alone. Therefore, in the presence of $50 \mu \mathrm{M}$ ATP and $1 \mathrm{mM}$ ADP, HS-AFM presumably detected $\mathrm{S} 1 \cdot \mathrm{ADP}$ bound to actin filaments before the low concentration of ATP in the presence of excess ADP slowly disrupted the binding. The decrease in the number of detected S1 molecules caused by Rng2CHD can be interpreted in the following two ways: (1) the number of transient binding events of S1 decreased, or (2) the duration of each binding event was shortened. Hypothesis (1) predicts that actin-activated S1 ATPase is also very strongly inhibited by Rng2CHD, which was not the case. We thus concluded that the unstable binding of S1•ADP to actin filaments caused by Rng2CHD shortened the duration of transient binding of S1 to actin filaments, and decreased the efficiency of detection of transient binding by HS-AFM. Rng2CHD decreased the fluorescence of HMM-GFP along actin filaments, a result that is consistent with this idea (Fig. 7A).

Based on this conclusion, we propose two possible mechanisms of acto-myosin II movement inhibition caused by Rng2CHD, in the framework of the swinging lever arm model (24, $25,26,27)$ tightly coupled with the acto-myosin ATPase cycle (28). The first mechanism proposes that phosphate release from myosin $\mathrm{I} \cdot \mathrm{ADP} \bullet \mathrm{Pi}$ is promoted normally even by actin filaments 
structurally altered by Rng2CHD, but without the lever arm swing. Consequently, myosin II•ADP, which does not have the authentic post-power stroke structure, reduces its affinity to actin filaments.

The second mechanism assumes that although the lever arm swing occurs, the myosin II motor domain slips at the contact surface with actin filaments, or myosin II•ADP dissociates from actin filaments because of the low affinity between myosin II•ADP and structurally altered actin filaments. This would lead to a failure of myosin II•ADP to maintain the tension, generated by the swing of the lever arm, to drive the movement of the actin filaments.

It is noteworthy that Rng2CHD did not inhibit actin filament movement on myosin V HMM, which is well established to move actin filaments slowly and processively with a lever arm swing (29, 30). In line with this finding, two actin mutations, M47A (31) and G146V (32), both of which likely perturb conformational changes of actin filaments, inhibit actin movements on myosin II, but not on myosin V. We suggest that the movement by myosin II, which is a fast and non-processive motor, requires some unidentified structural property of actin filaments which is dispensable for the slow and processive myosin $\mathrm{V}$ involving the lever arm swing. Notably, sparse binding of G680V mutant S1 along actin filaments accelerates the sliding movement on HMM by a factor of 2 (33). Since the $\mathrm{A} \cdot \mathrm{M} \bullet \mathrm{ADP} \cdot \mathrm{Pi}$ and $\mathrm{A} \cdot \mathrm{M} \bullet \mathrm{ADP}$ intermediates accumulate in the ATPase cycle of G680V S1 and actin filaments $(34,35)$, we speculated that certain positive cooperative conformational changes propagated from the sparsely distributed $\mathrm{A} \bullet \mathrm{M} \bullet \mathrm{ADP} \bullet \mathrm{Pi}$ or $\mathrm{A} \bullet \mathrm{M} \bullet \mathrm{ADP}$ complexes along the filament, resulting in 
the faster movement. It is tempting to speculate that Rng2CHD disrupts such positive cooperative conformational changes along actin filaments, assuming that they are required for fast and nonprocessive movement by myosin II. Further studies are needed to understand the mechanism by which actin filaments structurally altered by Rng2CHD fails to move on myosin II.

Another interesting feature of the inhibition of acto-myosin II movement by Rng2CHD is that there is hysteresis in the inhibition. The number of S1 molecules transiently bound to actin filaments in the presence of ATP and ADP was significantly reduced when S1 was added after Rng2CHD was allowed to interact with actin filaments (Fig. 8C). In contrast, when S1 was strongly and densely bound to actin filaments in the nucleotide-free state and then Rng2CHD was added together with ATP and ADP, Rng2CHD hardly changed the number of S1 molecules transiently bound to actin filaments (Fig. 8C). These results showed apparent hysteresis in the impact of Rng2CHD on S1 binding to actin filaments. We speculate that the dense rigor binding of S1 to actin filaments or the subsequent active interaction of S1 with actin induced by the addition of ATP changed the structure of actin filaments in one way, which hindered the subsequent inhibitory interaction of Rng2CHD with the altered actin filaments. This explanation is apparently at odds with the fact that Rng2CHD inhibited actomyosin movement in in vitro motility assays, in which myosin II HMM was bound to actin filaments in the rigor state before the addition of ATP and Rng2CHD. This discrepancy may be attributed to differences in experimental conditions, such as the density of the bound myosin heads 
along the actin filaments before the addition of Rng2CHD and ATP, or whether S1 molecules were present in the buffer containing ATP and Rng2CHD.

Phosphorylation of the myosin light chain $(36,37,38)$ and calcium regulation via tropomyosin and troponin $(39,40)$ are two widely known major regulatory mechanisms of actomyosin II movements. Additionally, it has been reported that caldesmon and calponin inhibit the movement of actin filaments on smooth muscle myosin II (41). These ABPs are similar to Rng2CHD in that they potently inhibit acto-myosin II movements even with sparse binding to actin filaments (41). Furthermore, calponin is homologous to Rng2CHD. However, these two ABPs are clearly different from Rng2CHD in their degree of cooperativity of motility inhibition (41) and ATPase inhibition $(42,43)$. In addition, some filaments move even in the presence of concentrations of caldesmon and calponin that stop the movement of most actin filaments (41), which is in sharp contrast to Rng2CHD that completely stops the movement of all actin filaments (Fig. 1A). It is unknown whether the inhibition of acto-myosin movement caused by these ABPs share the same mechanism with Rng2CHD, but Rng2CHD induces the highest degree of cooperative inhibition, making Rng2CHD suitable for elucidating the mechanism of acto-myosin II movement inhibition.

Further investigations are warranted to reveal the structural aspects of the acto-myosin II movement inhibition induced by Rng2CHD, and to clarify the relationship between the structural changes of actin filaments and acto-myosin II motility. The physiological significance of the potent 
inhibitory effect of Rng2CHD on acto-myosin II is another unresolved issue. A previous truncation study showed that $S$. pombe cells expressing mutant Rng2 lacking the CHD are able to contract CRs at normal rates (44). However, it is possible that both Rng2CHD and $\alpha$-actinin or some other ABP act as the regulator in a functionally redundant manner, in which case the knockout of either one would have no phenotype. Molecular and cell biological studies are also needed to understand the physiological role of Rng2CHD in vivo. 


\section{Materials and Methods}

\section{Protein purification}

Actin was purified from rabbit skeletal muscle acetone powder $(45,46)$. Myosin II HMM

and myosin II S1 were prepared by digestion of rabbit skeletal muscle myosin with papain and $\alpha$ chymotrypsin, respectively (47). HMM-GFP was expressed in D. discoideum and purified as described previously (13). The HMM version of human myosin V with a FLAG-tag at the N-terminus and a cmyc tag at the C-terminus was coexpressed with calmodulin in insect cells and purified using a method described previously (48).

The gene encoding Rng2CHD (12) was inserted at the HindIII and PstI sites of the pColdTEV vector (18), which had a TEV protease recognition sequence between the $6 \times$ His sequence and the multiple cloning site of pColdI (Takara Bio). The amino acid sequence of His-TEV Rng2CHD was MNHKVHHHHHHIEGRHMENLYFQGTLEGSEFKLDVNVGL...(Rng2CHD)...LPNFKA, where the underline shows the TEV recognition sequence.

Rng2CHD was expressed in BL21 Escherichia coli (Takara) according to the instructions provided by the manufacturer of pColdI. The cells were lysed by sonication in $2 \mathrm{mM} 2-$ mercaptoethanol, $0.3 \%$ Triton X-100, $0.1 \mathrm{mM}$ phenylmethylsulfonylfluoride, $400 \mathrm{mM} \mathrm{NaCl,} 10 \mathrm{mM}$ imidazole (pH 7.4) and $20 \mathrm{mM}$ Hepes ( $\mathrm{pH} 7.4$ ) on ice. The homogenate was clarified by centrifugation and mixed with Ni Sepharose 6 Fast Flow (GE Healthcare). After extensive washing, the peak 
fractions eluted by $7 \mathrm{mM}$ 2-mercaptoethanol, $400 \mathrm{mM}$ imidazole (pH 7.4) and $10 \mathrm{mM}$ Hepes (pH 7.4)

were combined and supplemented with His-tagged TEV protease at a 1/10 molar amount of proteins

in the pooled fractions to separate the His-tag from Rng2CHD at the cleavage site for TEV protease.

After dialysis against $50 \mathrm{mM} \mathrm{KCl}, 0.1 \mathrm{mM}$ DTT and $10 \mathrm{mM}$ Hepes (pH 7.4) overnight at $4^{\circ} \mathrm{C}$, the protein solution was clarified by centrifugation and passed through Ni Sepharose 6 Fast Flow in a column to remove the released His-tag, His-TEV protease and uncleaved His-tagged Rng2CHD. This was followed by concentration with a centrifugal concentrator (Amicon Ultra-15 $3 \mathrm{k}$ device, Merck Millipore), and after supplemented with $10 \mathrm{mM}$ DTT, aliquots were snap-frozen in liquid nitrogen and stored at $-80^{\circ} \mathrm{C}$.

To fuse GFP to the N-terminus of Rng2CHD, the GFP gene with a S65T mutation was inserted at the KpnI and BamHI sites of pCold-TEV-Rng2CHD. A Gly-based 16 amino acid-residue linker sequence was inserted between the GFP gene and the Rng2CHD gene, so that the expressed GFP would not spatially inhibit the binding of Rng2CHD to the actin filament. The resultant amino acid sequence

of GFP-Rng2CHD was MNHKVHHHHHHIEGRHMENLYFQGTMSKGE...(GFP)...MDELYGGSEFGSSGSSGSSKLDV NVGL...(Rng2CHD)...LPNFKA, where the underline shows the TEV recognition sequence and the double underline shows the linker sequence. GFP-Rng2CHD was expressed in Rosetta DE3 E. coli (Merck Millipore), and purified basically in the same way as Rng2CHD, except that the protein was 
further purified by anion exchange chromatography with an Econo-pac High Q cartridge (BIO-RAD)

before it was concentrated.

\section{In vitro motility assays}

G-actin was polymerized in F-buffer $(0.1 \mathrm{M} \mathrm{KCl}, 2 \mathrm{mM} \mathrm{MgCl} 2,1 \mathrm{mM}$ DTT, $1 \mathrm{mM}$ ATP

and $10 \mathrm{mM}$ Hepes pH 7.4) for $1 \mathrm{~h}$ at $22^{\circ} \mathrm{C}$, and was diluted to $1 \mu \mathrm{M}$ with $\mathrm{NF}$ buffer $(25 \mathrm{mM} \mathrm{KCl}, 2$ $\mathrm{mM} \mathrm{MgCl} 2,10 \mathrm{mM}$ DTT and $20 \mathrm{mM}$ Hepes $\mathrm{pH}$ 7.4). Diluted actin filaments were incubated with 1 $\mu \mathrm{M}$ rhodamine phalloidin (Invitrogen) or $1 \mu \mathrm{M}$ phalloidin (Wako) for $1 \mathrm{~h}$ at $22^{\circ} \mathrm{C}$.

In vitro actomyosin motility assays in which actin filaments move on myosin II were performed according to the method of Kron and Spudich (7). Rhodamine phalloidin-stabilized actin filaments were bound to myosin II HMM on a nitrocellulose-coated glass surface in a flow chamber filled with NF buffer containing $10 \mathrm{mg} / \mathrm{ml}$ BSA. Movement of actin filaments was started by injecting $15 \mu \mathrm{l}$ of MA buffer ( $25 \mathrm{mM} \mathrm{KCl}, 2 \mathrm{mM} \mathrm{MgCl} 2,0.5 \mathrm{mM}$ ATP, $10 \mathrm{mM}$ DTT, $3 \mathrm{mg} / \mathrm{ml}$ glucose, $1.2 \mu \mathrm{M}$ glucose oxidase, $0.15 \mu \mathrm{M}$ catalase, $10 \mathrm{mg} / \mathrm{ml}$ BSA and $20 \mathrm{mM}$ Hepes $\mathrm{pH}$ 7.4) into the flow chamber. Various concentrations of Rng2CHD (5, 10, 50, 100 and $200 \mathrm{nM})$ were present in this buffer. Fluorescence of rhodamine phalloidin was imaged with an EMCCD camera (iXon X3, Andor) on a fluorescent microscope (IX-71; Olympus) equipped with a Plan Apo 100X, 0.9 NA objective lens (Nikon) at a frame rate of $0.5 \mathrm{fps}$. 
The acto-myosin II motility assay in the presence of GFP-Rng2CHD was performed basically as above, with several modifications. Rhodamine phalloidin stabilized and unlabeled phalloidin stabilized actin filaments were mixed at a 1:1 concentration in order to observe GFP fluorescence without the interference of rhodamine fluorescence. Fluorescence micrographs were taken with an EMCCD camera (iXon X3) on a TIRF microscope (IX-71) equipped with a UApo N 100X, 1.49 NA objective lens (Olympus). Laser light of $538 \mathrm{~nm}$ for exciting rhodamine and $488 \mathrm{~nm}$ for exciting GFP were irradiated alternately at $2 \mathrm{~s}$ intervals, and the time-lapse imaging of moving actin filaments and GFP-Rng2CHD dynamics was performed semi-simultaneously.

The images were processed with Image J (49). Movements of actin filaments were tracked by MTrackJ, a plugin for Image J (50) and the velocities were calculated. The fluorescence intensity of GFP was quantified by Image $\mathbf{J}$ as follows. Five filaments near the center of the images were selected for each condition, and the fluorescence intensity in five frames was measured. The light intensity at five points near the filament was averaged and subtracted from the measured values along the filament as the background for each filament. The values were normalized by the unit length of each filament.

For in vitro motility assays in which actin filaments move on myc-tagged myosin V HMM, HMM molecules were immobilized on a glass surface via anti-c-myc antibody. MA2 buffer ( $20 \mathrm{mM}$ $\mathrm{KCl}, 4 \mathrm{mM} \mathrm{MgCl}$, 2 mM ATP, $120 \mu \mathrm{M}$ calmodulin, 1 mM EGTA, 1 mM DTT, 0.5\% methylcellulose 
(v/v), $1 \mathrm{mg} / \mathrm{ml} \mathrm{BSA}$ and $25 \mathrm{mM}$ imidazole $\mathrm{pH}$ 7.4) was used instead of MA buffer. Mouse calmodulin was expressed in E. coli and purified as described previously (51). The fluorescence images were captured with a camera (ORCA-Flash 2.8; Hamamatsu Photonics) on a fluorescent microscope (IX70; Olympus) equipped with a Plan-Fluor 100X, 1.3 NA objective lens (Nikon) at a frame rate of 0.5 fps.

\section{Measurement of dissociation constant}

G-actin was polymerized in F-buffer for $1 \mathrm{~h}$ at $22^{\circ} \mathrm{C}$ and incubated with phalloidin at a $1: 1$

molar ratio for $1 \mathrm{~h}$ at $22^{\circ} \mathrm{C}$. Phalloidin-stabilized actin filaments that were diluted to $3 \mu \mathrm{M}$ and various concentrations of Rng2CHD or GFP-Rng2CHD $(1,2,3,4,5,6 \mathrm{nM})$ were incubated together in SA buffer ( $25 \mathrm{mM} \mathrm{KCl}, 2 \mathrm{mM} \mathrm{MgCl}, 0.5 \mathrm{mM} \mathrm{ATP}, 10 \mathrm{mM}$ DTT and $20 \mathrm{mM}$ Hepes $\mathrm{pH}$ 7.4) for $5 \mathrm{~min}$ at $22^{\circ} \mathrm{C}$, and then centrifuged at $278,800 \mathrm{~g}$ for $10 \mathrm{~min}$ at $22^{\circ} \mathrm{C}$. The supernatants and pellets were subjected to SDS-PAGE. Images of Coomassie brilliant blue (CBB)-stained gels were read into Image $\mathrm{J}$ and the concentration of Rng2CHD in each fraction was quantified by densitometry. The dissociation constant $\left(K_{d}\right)$ for Rng2CHD to actin filaments was calculated by fitting plots of [Rng2CHD bound to actin filaments] versus [Rng2CHD free] with the following equation:

$$
\left[\text { Rng2CHD } D_{\text {bound }}\right]=\left[\text { Actin }_{\text {total }}\right][\text { Rng2CHD free }] /\left(\left[\text { Rng2CHD free }+K_{d}\right]\right)
$$

$K_{d}$ between actin filaments and GFP-Rng2CHD was calculated in the same way. 


\section{Estimation of binding ratio of Rng2CHD and GFP-Rng2CHD to actin filament from $K_{d}$}

$K_{d}$ between Rng2CHD and actin filaments is given by:

$$
K_{d}=\left[\text { Actin }_{\text {free }}\right]\left[\operatorname{Rng} 2 C H D_{\text {free }}\right] /[\text { Rng2CHD } \text { bound }]
$$

The concentration of actin filaments is extremely low in solutions in which Rng2CHD in the buffer interacts with actin filaments immobilized on the substrate, such as in vitro actomyosin motility assays and observations of binding with high-speed AFM and fluorescence microscopy, so that $\left[R n g 2 C H D_{\text {free }}\right]$ can be approximated by the concentration of total Rng2CHD ([Rng2CHD total $])$. Therefore, the following approximation holds from Eq. 2:

$$
[\text { Rng2CHD } \text { bound }] /\left[\text { Actin }_{\text {free }}\right] \simeq[\text { Rng2CHD } \text { total }] / K_{d}
$$

The binding ratios of Rng2CHD and GFP-Rng2CHD to the dilute actin protomers were estimated with this approximation using the value of $K_{d}$.

\section{Electron microscopy}

G-actin was polymerized in F-buffer for $1 \mathrm{~h}$ at room temperature, and $1 \mu \mathrm{M}$ actin filaments were mixed with various concentrations of Rng2CHD in EM buffer $(25 \mathrm{mM} \mathrm{KCl}, 4 \mathrm{mM} \mathrm{MgCl} 2,1$ mM DTT, $0.1 \mathrm{mM}$ ATP and $10 \mathrm{mM}$ imidazole $\mathrm{pH}$ 7.4) at room temperature. A small volume of each sample was placed on a carbon-coated grid at $30 \mathrm{~s}$ (40 nM Rng2CHD), 2 min (200 nM Rng2CHD) or 4 min ( $1 \mu \mathrm{M}$ Rng2CHD) after mixing. The samples were negatively stained with $1 \%$ uranyl acetate, 
and observed in a transmission electron microscope (Tecnai F20; FEI). The electron micrographs were

recorded with a Gatan ORIUS 831 CCD camera, adjusted for contrast and Gaussian-filtered using

Adobe Photoshop.

\section{Myosin II S1 ATPase measurements}

Actin-activated S1 ATPase was measured according to the method of Kodama et al. using

malachite green (52). G-actin was polymerized in F-buffer for $1 \mathrm{~h}$ at $22^{\circ} \mathrm{C}$. The solution was centrifuged at $278,800 \mathrm{~g}$ for $10 \mathrm{~min}$ at $22^{\circ} \mathrm{C}$ and actin filaments in the pellet were resuspended in $\mathrm{NF}$

buffer. This procedure was repeated once more to minimize the amount of carried over phosphate.

Actin filaments diluted to $23.8 \mu \mathrm{M}$ was mixed with various concentrations of Rng2 $\mathrm{CHD}(0,0.33,0.82$,

$1.91,5 \mu \mathrm{M})$ in NF buffer containing $2 \mathrm{mM} \mathrm{ATP}$, and was incubated for $10 \mathrm{~min}$ at $25^{\circ} \mathrm{C}$. The reaction was started by the addition of $50 \mathrm{nM} \mathrm{S1}$, and phosphate released at $0,2,4,6$ and 8 min was measured.

\section{Co-sedimentation assay of actin filaments and S1 with Rng2CHD}

For the co-sedimentation assays in the presence of ATP, G-actin was polymerized in F-buffer

for $1 \mathrm{~h}$ at $22^{\circ} \mathrm{C}$ and was then incubated with phalloidin at a $1: 1$ molar ratio for $1 \mathrm{~h}$ at $22^{\circ} \mathrm{C}$. For the cosedimentation assays in the presence of ADP, the solution of actin filaments was centrifuged at 278,800 g for $10 \mathrm{~min}$ at $22^{\circ} \mathrm{C}$, and the pelleted actin filaments were resuspended in NF buffer. This procedure was repeated once again to minimize the amount of carried over ATP before the addition of phalloidin. 
The following two samples were prepared for both experiments: (1) $3 \mu \mathrm{M}$ actin filaments were incubated with $2 \mu \mathrm{M} \mathrm{S} 1$ for $5 \mathrm{~min}$ at $22^{\circ} \mathrm{C}$; (2) $3 \mu \mathrm{M}$ actin filaments were incubated with Rng2CHD for $10 \mathrm{~min}$ at $22^{\circ} \mathrm{C}$, and then incubated with subsequently added $\mathrm{S} 1$ for 5 min at $22^{\circ} \mathrm{C}$. Each sample was prepared in S-ATP buffer $\left(25 \mathrm{mM} \mathrm{KCl}, 2 \mathrm{mM} \mathrm{MgCl}_{2}, 2 \mathrm{mM}\right.$ ATP, $10 \mathrm{mM}$ DTT, $10 \mathrm{mM}$ phosphoenolpyruvate, 10 units / $\mathrm{ml}$ pyruvate kinase and $20 \mathrm{mM}$ Hepes $\mathrm{pH}$ 7.4) or S-ADP buffer (25 $\mathrm{mM} \mathrm{KCl}, 2 \mathrm{mM} \mathrm{MgCl} 2,2 \mathrm{mM}$ ADP, $10 \mathrm{mM}$ DTT and $20 \mathrm{mM}$ Hepes $\mathrm{pH}$ 7.4). After incubation, each sample was centrifuged at $278,800 \mathrm{~g}$ for $10 \mathrm{~min}$ at $22^{\circ} \mathrm{C}$. The supernatants and pellets were subjected to SDS-PAGE. The images of the CBB-stained gels were read into Image $\mathrm{J}$ and the concentration of $\mathrm{S} 1$ in each fraction was quantified by densitometry.

\section{Fluorescence microscope-based binding assay}

Binding of HMM-GFP to actin filaments was observed as follows. G-actin was polymerized in NF-buffer $(50 \mathrm{mM} \mathrm{KCl}, 2 \mathrm{mM} \mathrm{MgCl} 2,0.5 \mathrm{mM}$ EGTA, $1 \mathrm{mM}$ DTT, $20 \mathrm{mM}$ PIPES, $\mathrm{pH}$ 6.5) containing $0.2 \mathrm{mM}$ ATP for $2 \mathrm{~h}$ at $22^{\circ} \mathrm{C}$. Actin filaments and Alexa 647 phalloidin (Invitrogen) were mixed at a molar ratio of 20:1 and incubated overnight on ice. The surface of coverslips was covered with a positively charged lipid bilayer and was used to construct flow chambers as described previously (18), except that the weight ratio of 1,2-dipalmitoyl-sn-glycero-3-phosphocholine (DPPC; Avanti Polar Lipids, Alabaster, AL) and 1,2-dipalmitoyl-3-trimethylammonium-propane (DPTAP; 
Avanti Polar Lipids) was 17:3 (14). Alexa 647 phalloidin-stabilized actin filaments diluted in NF-ATP

buffer (NF buffer containing $0.5 \mu \mathrm{M}$ ATP) were introduced into the flow chamber to loosely bind on the positively charged lipid monolayer. HMM-GFP and Rng2CHD diluted in NF-ATP buffer were then introduced to the flow chamber. Alternatively, NF-ATP buffer in the above procedures was replaced with NF buffer for the assays in the nucleotide-free state. Fluorescence of Alexa 647 and GFP was imaged with a fluorescence microscope (ECLIPSE E600, Nikon, Tokyo, Japan) equipped with an ARUGUS-HiSCA system (Hamamatsu Photonics, Hamamatsu, Japan). Images were captured using a 100x objective lens (CFI Plan Apo Lambda 100x Oil, NA 1.45; Nikon).

\section{High-speed atomic force microscopy}

We used a laboratory-built high-speed atomic microscope (HS-AFM) as described previously (53). The cantilevers and the sample stage, positively charged lipid bilayers on a mica surface, were prepared according to the method of Ngo et al. (18). The free oscillation amplitude of the cantilever was set at $2.0 \mathrm{~nm}$, and the amplitude set point for feedback control was set to $1.6-1.9$ nm during observations.

G-actin was polymerized in F-buffer for $1 \mathrm{~h}$ at $22^{\circ} \mathrm{C}$, and then the buffer on the sample stage was replaced by $2 \mu \mathrm{l}$ of $20 \mu \mathrm{M}$ actin filaments in AFM buffer ( $25 \mathrm{mM} \mathrm{KCl,} 2 \mathrm{mM} \mathrm{MgCl} 2,50$ $\mu \mathrm{M}$ ATP, $1 \mathrm{mM}$ ADP, $10 \mathrm{mM}$ DTT, $20 \mathrm{mM}$ Hepes $\mathrm{pH}$ 7.4). After incubation for $10 \mathrm{~min}$ at $22^{\circ} \mathrm{C}$, the 
surface of the sample stage was rinsed with $20 \mu \mathrm{l}$ of AFM buffer to remove free actin filaments.

Subsequently, the surface of the sample stage was immersed in $60 \mu 1$ of AFM buffer in the observation chamber of the HS-AFM. The observations of the transient binding of myosin II S1 to actin filaments in the presence of ATP and ADP were performed under the following three conditions: (1) S1 diluted in AFM buffer was added to the observation chamber to a final concentration of $500 \mathrm{nM}$; (2) $12.4 \mathrm{nM}$ Rng2CHD was allowed to interact with actin filaments in the observation chamber for $15 \mathrm{~min}$ at $22^{\circ} \mathrm{C}$, and then $20 \mu \mathrm{M} \mathrm{S1}$ in AFM buffer was added to the observation chamber to a final concentration of $500 \mathrm{nM}$; (3) $500 \mathrm{nM} \mathrm{S1}$ was allowed to interact with actin filaments in the observation chamber filled with NF buffer ( $25 \mathrm{mM} \mathrm{KCl}, 2 \mathrm{mM} \mathrm{MgCl} 2,10 \mathrm{mM}$ DTT, $20 \mathrm{mM}$ Hepes pH 7.4) for $10 \mathrm{~min}$ at $22^{\circ} \mathrm{C}$. Subsequently, 1/40 vol of a mixture of ATP, ADP and Rng2CHD in AFM buffer was added to the observation chamber to a final concentration of $50 \mu \mathrm{M}, 1 \mathrm{mM}$ and $12.4 \mathrm{nM}$, respectively.

AFM images were obtained at a scan speed of $0.5 \mathrm{~s}$ per field of view, and were then visualized by Kodec4, our laboratory-built software (18). Images scanned between 1 min and 2 min after the addition of S1 or ATP were analyzed, and the number of transiently bound S1 molecules on actin filaments was visually counted. 


\section{Acknowledgments}

We thank Dr. Toshio Ando for technical support for HS-AFM experiments, and Dr. Atsuko

H. Iwane and Dr. Toshio Yanagida for the gift of the virus to express HMM of myosin V. This work

was supported in part by Bio-SPMs Collaborative Research of WPI Nano Life Science Institute,

Kanazawa University, and Grants-in-aid from the Ministry of Education, Culture, Sports, Science and

Technology to KT (No. 24370069 and 24117008), K Nakano (No. 22019004), MT (No.

24770177) and TU (No. 24370069 and 24117008).

\section{Author contributions}

NK, KT, ON, KN and TU conceived and supervised the study, YH, MT, KXN, KT, KN and

TU designed the experiments, YH, MT, TI, MY, KH and TU performed the experiments, YH, MT,

MY, KH and TU analyzed the data, YH and TU wrote the manuscript draft and $\mathrm{KH}, \mathrm{NK}, \mathrm{KT}$ and $\mathrm{KN}$

revised the manuscript.

\section{Conflict of interest}

The authors declare no conflict of interest. 


\section{Figures}

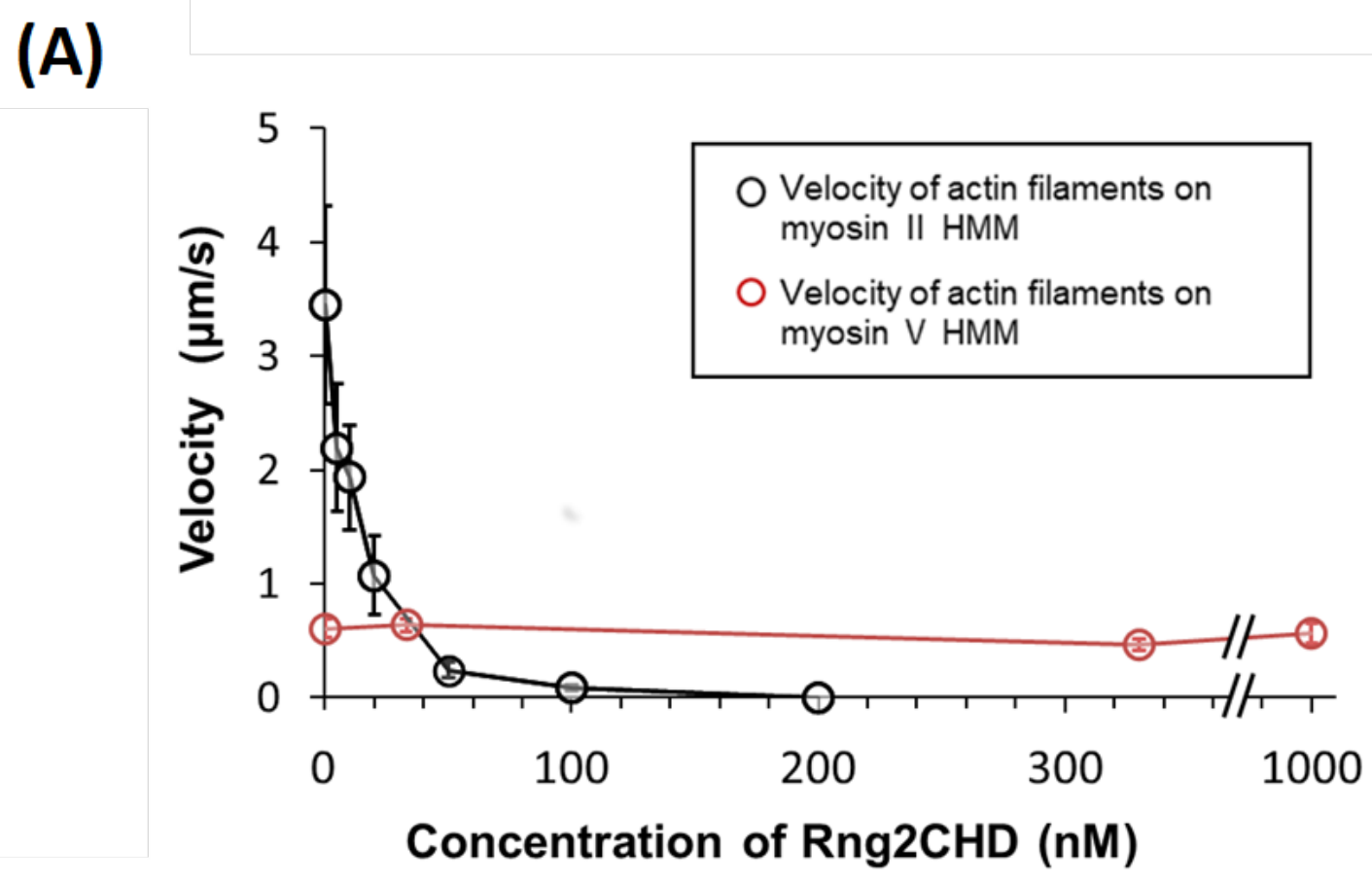

(B)

O nM Rng2CHD

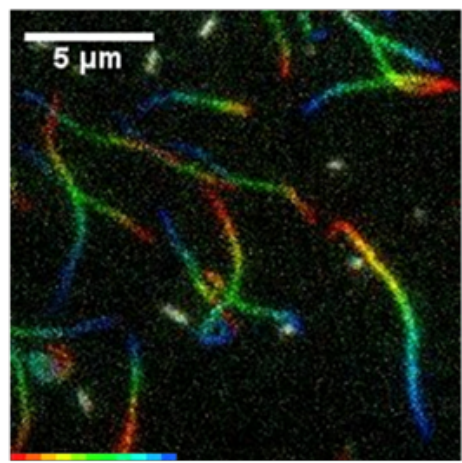

+5 nM Rng2CHD

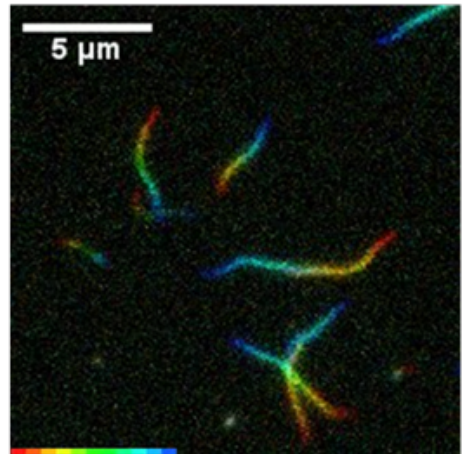

+50 nM Rng2CHD

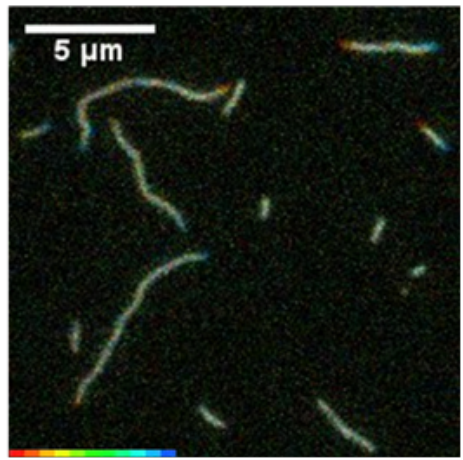

Figure 1 Rng2CHD strongly inhibits movement of actin filaments on myosin II, but not on

myosin V.

(A) Movement velocity of actin filaments on myosin II HMM (black plots) or myosin V HMM (red 

made available under aCC-BY-NC 4.0 International license.

plots) attached to glass surfaces in the presence of various concentrations of Rng2CHD. The concentration of ATP was $0.5 \mathrm{mM}$ for myosin II HMM, and $2 \mathrm{mM}$ for myosin V HMM. Ten smoothly moving filaments were chosen for each condition, and approximately 10 consecutive measurements were made for each filament. Data are expressed as the mean \pm SD. (B) Trajectories of moving actin filaments on myosin II HMM in the presence of 0, 5 and $50 \mathrm{nM}$ Rng2CHD. Eleven consecutive images with $0.2 \mathrm{~s}$ intervals are coded in rainbow colors from red to blue, and overlaid. 


\section{(A)}

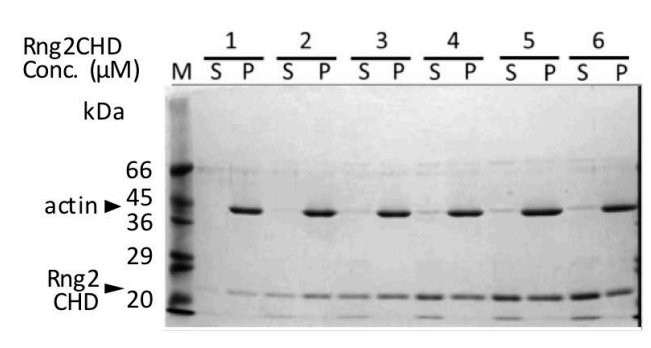

(B)

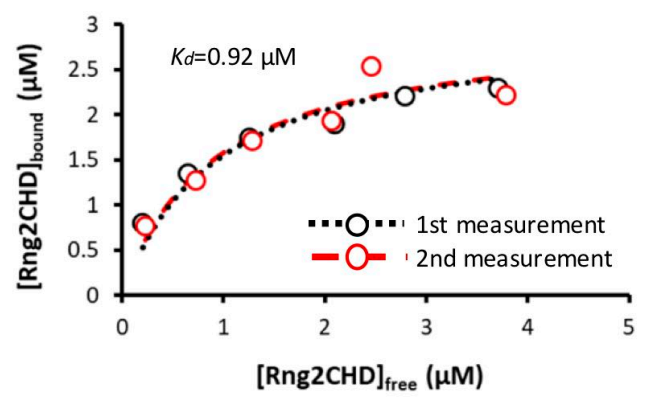

Figure 2 Measurement of $K_{d}$ between actin filaments and Rng2CHD or GFP-Rng2CHD.

(A) Co-sedimentation assay of Rng2CHD with $3 \mu \mathrm{M}$ actin filaments. (B) The concentration of bound

Rng2CHD was plotted against the concentration of the free fraction and fitted with the following

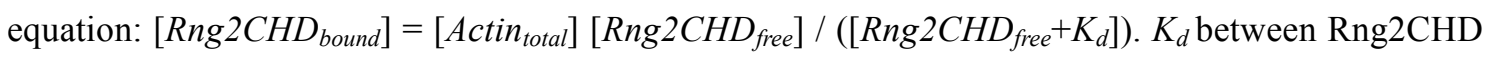
and actin protomers was calculated as an average value of the two trials. 


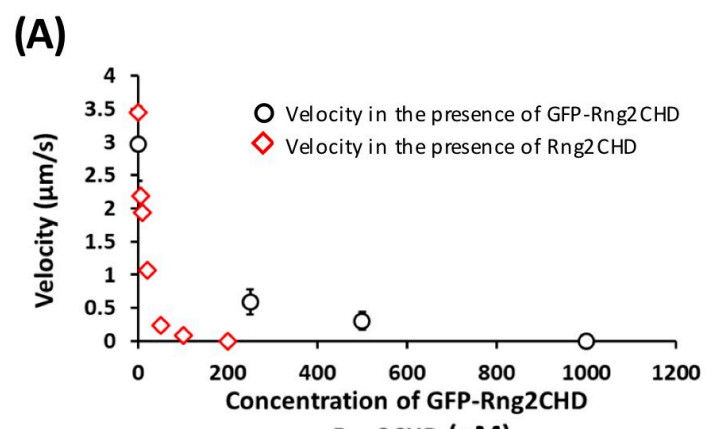

or Rng2CHD (nM)

(C)

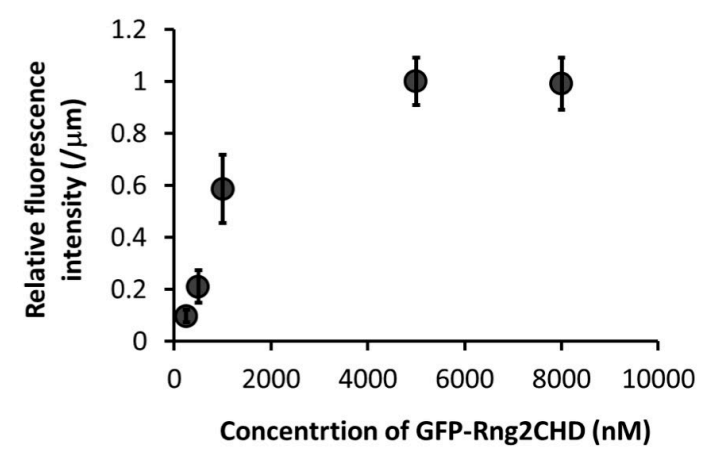

(B)

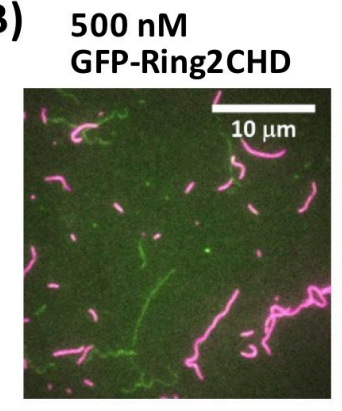

(D)

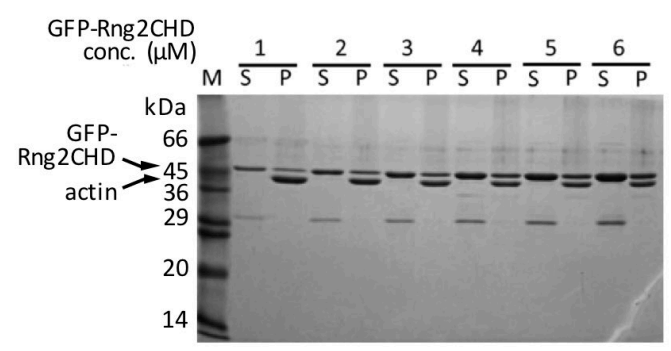

Figure 3 Sparsely bound GFP-Rng2CHD on actin filaments inhibits movement of actin filaments

on myosin II.

(A) Movement velocity of actin filaments on myosin II HMM-coated glass surfaces in the presence of various concentration of GFP-Rng2CHD (black plots). Ten smoothly moving filaments were chosen for each condition, and approximately 10 consecutive measurements were made for each filament.

Data are expressed as the mean $\pm \mathrm{SD}$. The movement velocity in the presence of Rng2CHD is also shown for the reference (red plots). (B) Fluorescence micrographs of GFP-Rng2CHD bound to actin filaments. Actin filaments stabilized by non-fluorescent phalloidin and those labeled with rhodamine phalloidin were present at a molar ratio of 1: 1. Green: GFP fluorescence. Red: rhodamine fluorescence. 
(green filaments). (C) Fluorescence intensity of GFP-Rng2CHD on non-fluorescent phalloidinstabilized actin filaments. The fluorescence intensity along five filaments was measured for five frames. Data are expressed as the mean \pm SD of 25 measurements. Bars: $10 \mu \mathrm{m}$. (D) Cosedimentation of GFP-Rng2CHD with $3 \mu \mathrm{M}$ actin filaments. Similar molar amounts of actin and GFP-Rng2CHD were recovered in the pellet fractions when [GFP-Rng2CHD] was 5 and $6 \mu \mathrm{M}$. The GFP-Rng2CHD preparation was contaminated by a bacterial $\sim 30 \mathrm{kDa}$ protein. 
bioRxiv preprint doi: https://doi org/10.1101/2020 04 14.041046: this version posted May 23,2020 . The copyright holder for this preprint (which was not certified by peer review) is the author/funder, who has granted bioRxiv a license to display the preprint in perpetuity. It is made available under aCC-BY-NC 4.0 International license.

(A) Control
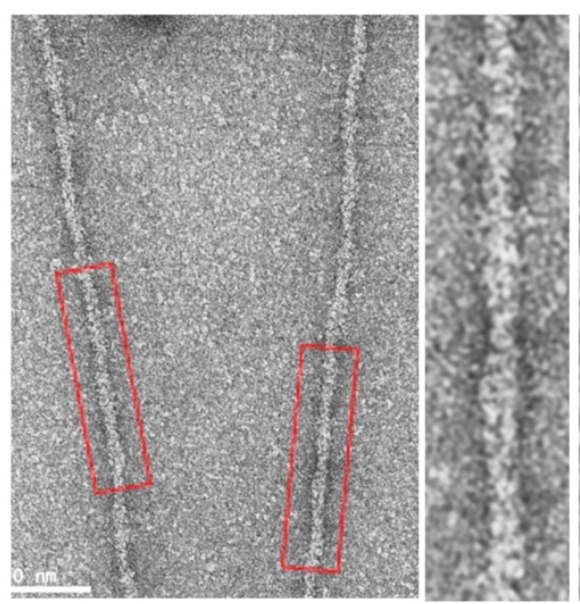

(C)

200 nM Rng2CHD

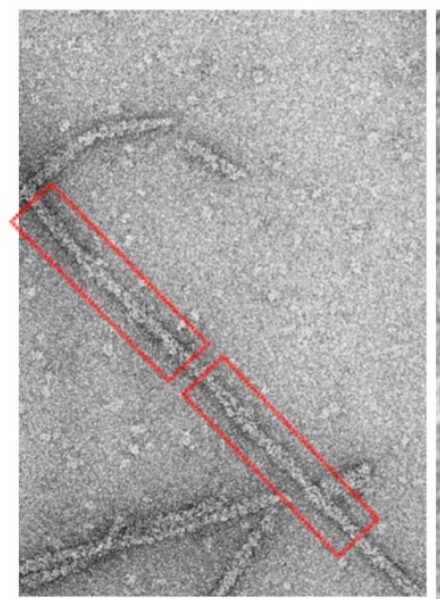

$50 \mathrm{~nm}$

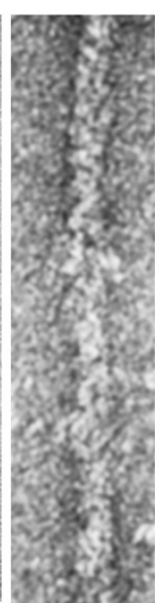

$10 \mathrm{~nm}$
(B) $\quad 40 \mathrm{nM}$ Rng2CHD
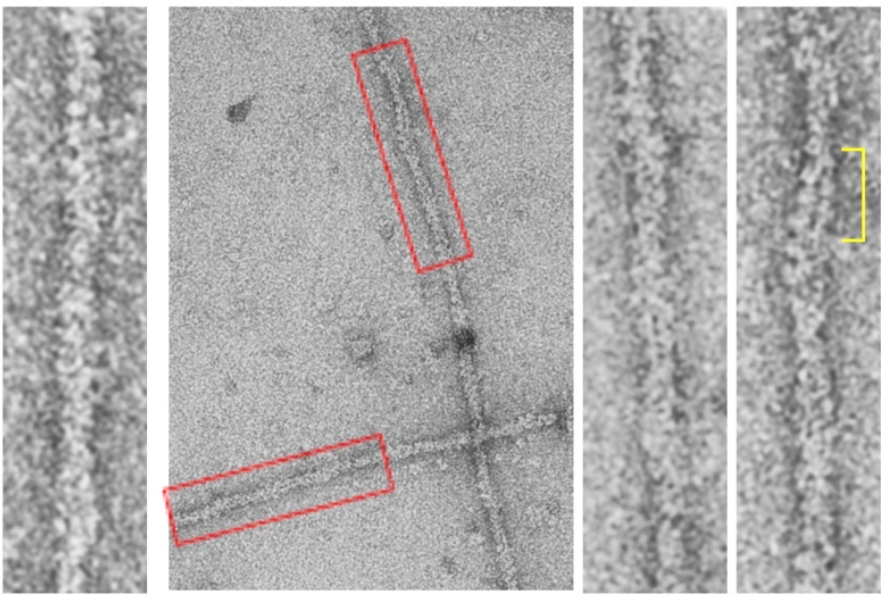

(D) $\quad 1 \mu \mathrm{M}$ Rng2CHD
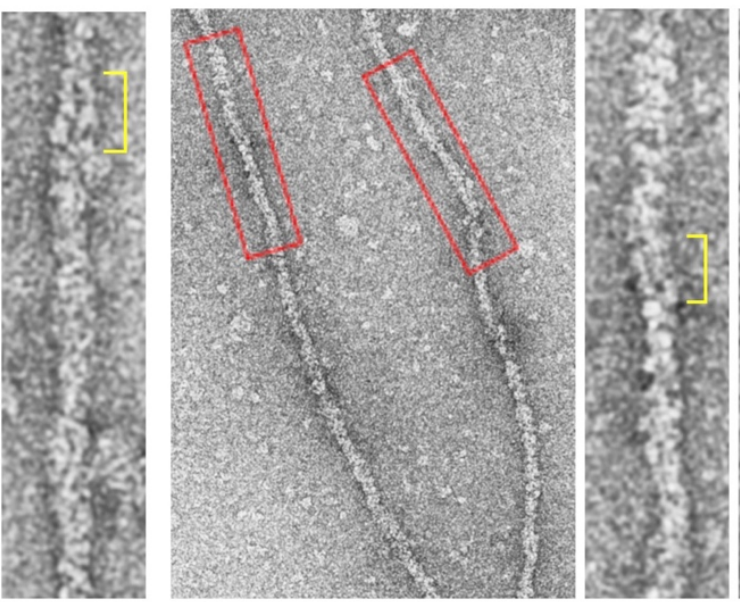

$50 \mathrm{~nm}$

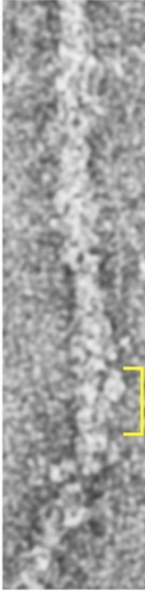

$10 \mathrm{~nm}$

Figure 4 Rng2CHD deforms the helical structures of actin filaments.

Electron micrographs of negatively stained actin filaments in the presence of $0 \mathrm{nM}(\mathrm{A}), 40 \mathrm{nM}(\mathrm{B})$,

$200 \mathrm{nM}$ (C) and $1 \mu \mathrm{M}$ (D) Rng2CHD. The boxed regions in each image are magnified to the right. In

the presence of Rng2CHD, the filaments often appear to be slightly curved or bent. In some parts, the

two actin protofilaments look separated (yellow brackets). At a high concentration of Rnd2CHD, the

filaments are highly deformed and show discontinuities (D). 


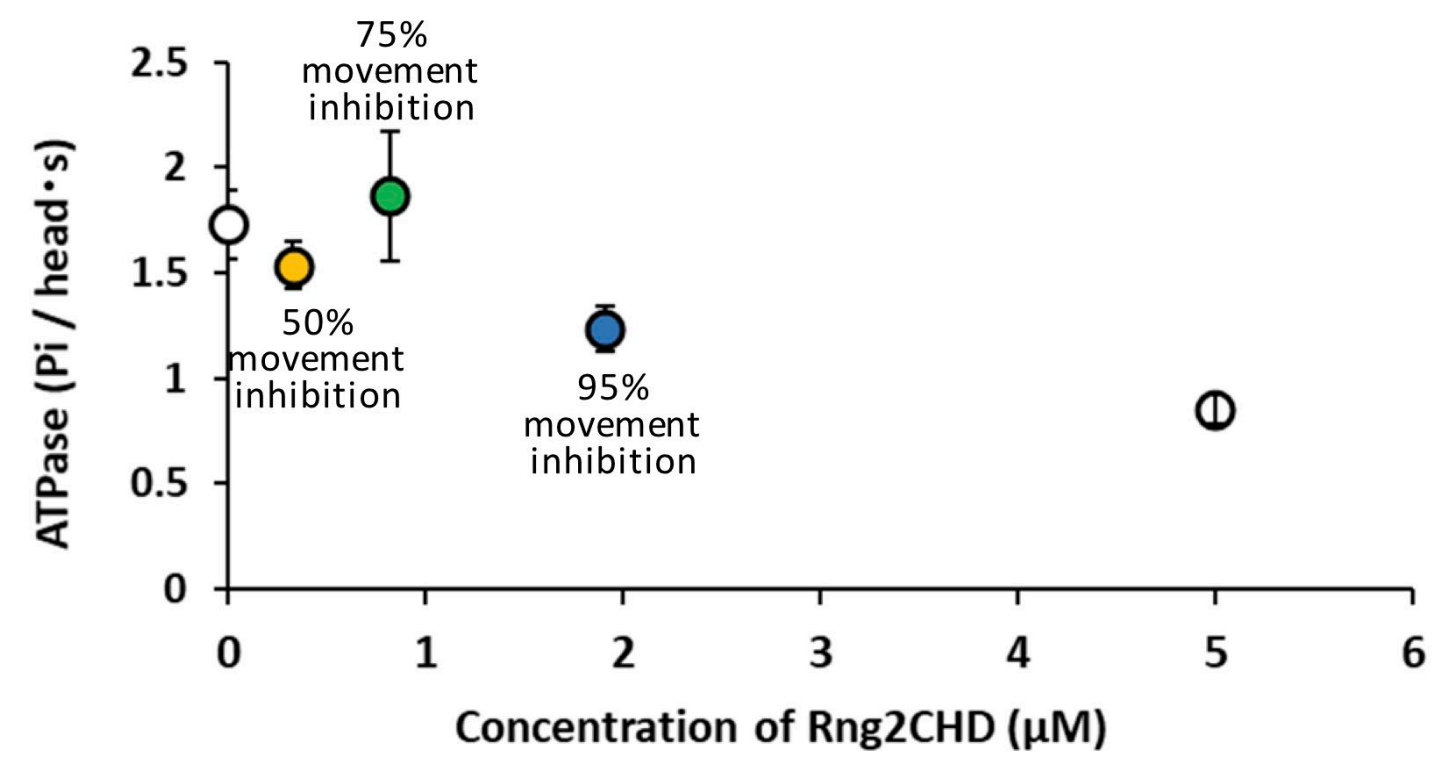

Figure 5 Actin-activated S1 ATPase in the presence of Rng2CHD.

The orange $(0.33 \mu \mathrm{M}$ Rng2CHD), green $(0.82 \mu \mathrm{M}$ Rng2CHD) and blue (1.91 $\mu \mathrm{M}$ Rng2CHD) plots were measured in the presence of Rng2CHD concentrations that were expected to bind to actin protomers at binding ratios which caused a $50 \%, 75 \%$ and $95 \%$ reduction of acto-myosin II movement speed in in vitro motility assays, respectively. Data are expressed as the mean $\pm \mathrm{SD}$ of three independent experiments. 


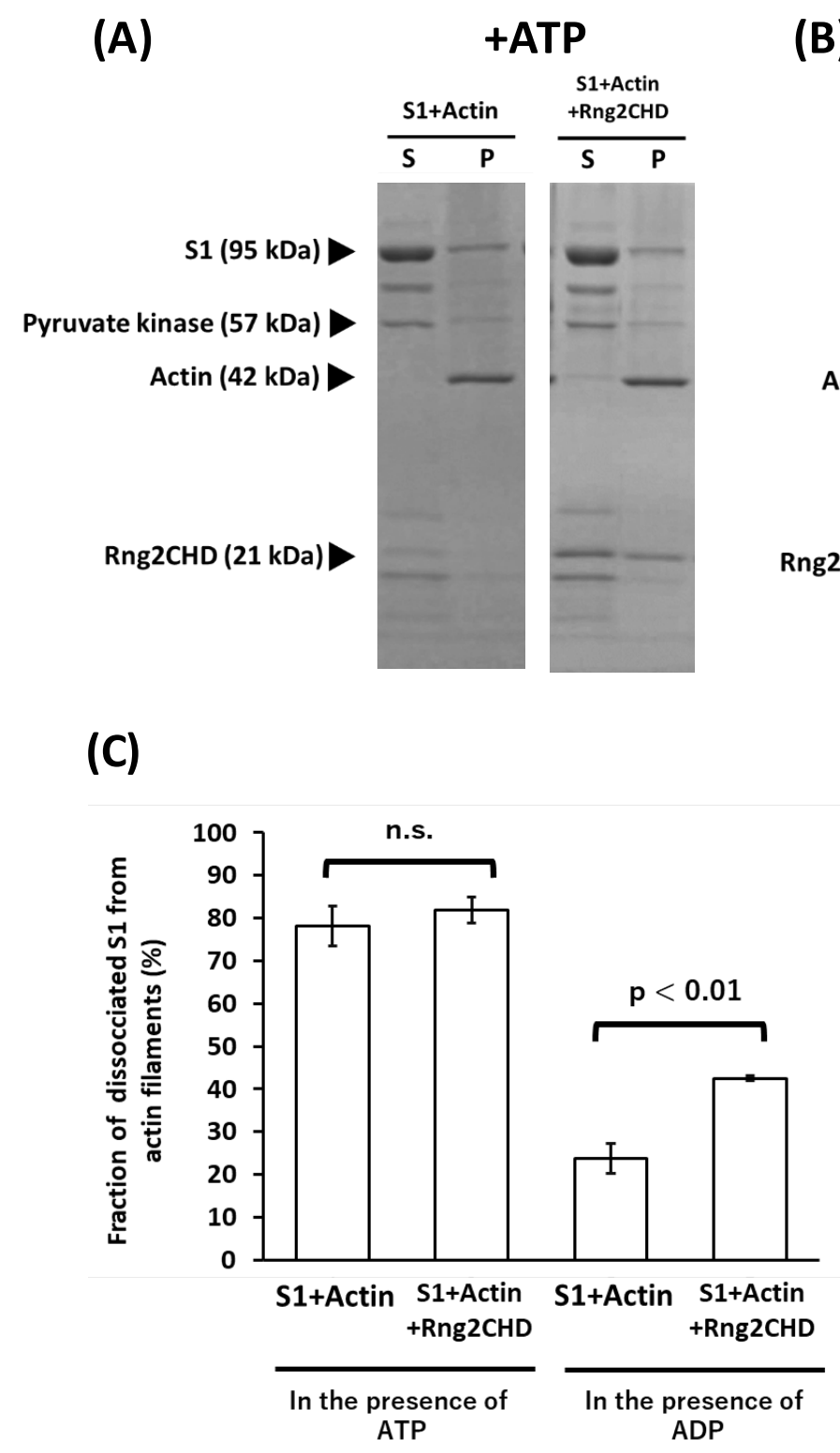

Figure 6 Rng2CHD inhibits the steady-state binding of S1 to actin filaments in the presence of

ADP, but not in the presence of ATP.

(A,B) Co-sedimentation assay of S1 and actin filaments in the presence of Rng2CHD and $2 \mathrm{mM}$ ATP

(A) or 2 mM ADP (B). (C) Fraction of S1 dissociated from actin filaments was compared with and without Rng2CHD. Rng2CHD made a significant difference in the presence of ADP (Student's t-test, 
bioRxiv preprint doi: https://doi.org/10.1101/2020.04.14.041046; this version posted May 23, 2020. The copyright holder for this preprint (which was not certified by peer review) is the author/funder, who has granted bioRxiv a license to display the preprint in perpetuity. It is made available under aCC-BY-NC 4.0 International license.

$\mathrm{p}<0.01$ ), but not in the presence of ATP. Data are expressed as the mean \pm SD of three independent experiments. 
(A) $+0.5 \mu \mathrm{M}$ ATP

Rng2CHD conc.
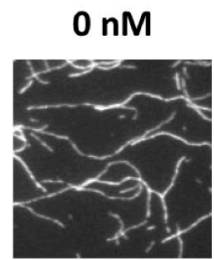

Actin fiaments

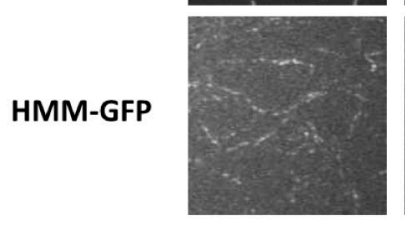

- ATP

(B)

$$
\text { Rng2CHD conc. }
$$

Actin fiaments
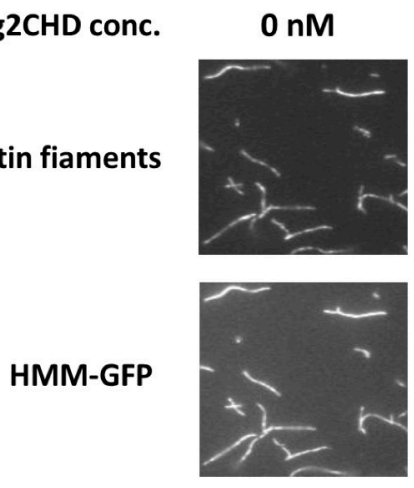

$$
1 \mathrm{nM}
$$
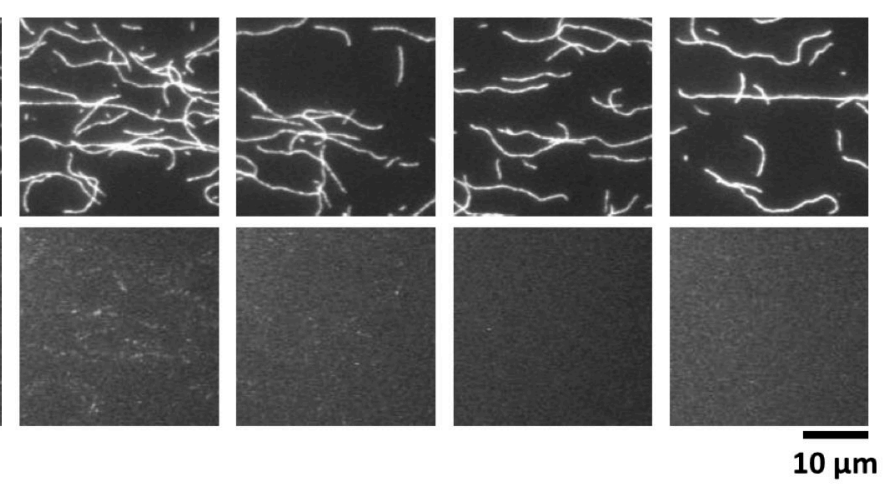

Figure 7 Rng2CHD decreases the fluorescence of HMM-GFP along actin filaments in the

presence of ATP.

Fluorescence micrographs of actin filaments labeled lightly with Alexa647 phalloidin and HMM-GFP

in the presence of $0.5 \mu \mathrm{M}$ ATP (A) and in the nucleotide-free state (B). In each panel, the top row

shows the fluorescence of Alexa647, and the bottom row shows that of GFP. 
(A)

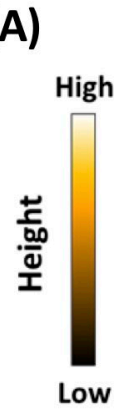

$+500 \mathrm{nM} \mathrm{S1}$

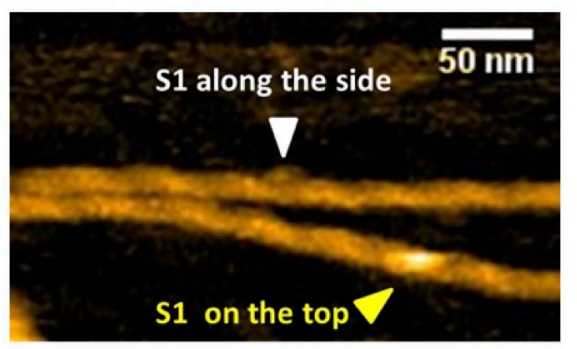

(B) $12.4 \mathrm{nM}$ Rng2CHD then $+500 \mathrm{nMS1}$

(C)

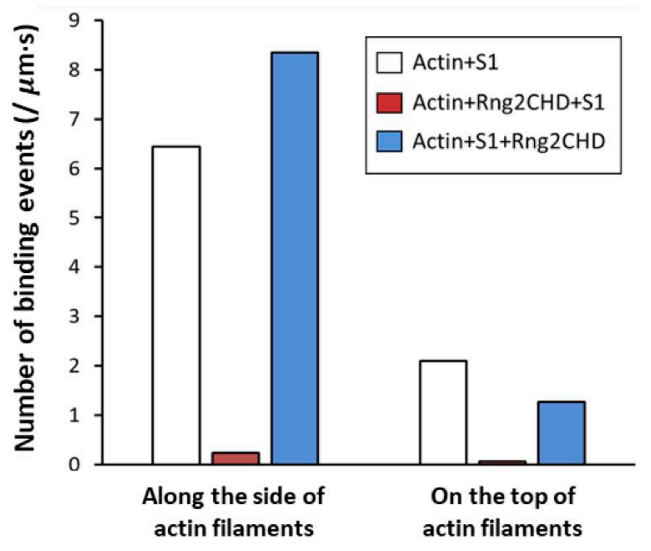

Figure 8 Rng2CHD significantly decreases the number of S1 molecules bound along actin

\section{filaments in the presence of ATP.}

(A, B) HS-AFM images of actin filaments interacting with S1 in the presence of $50 \mu \mathrm{M}$ ATP and 1

$\mathrm{mM}$ ADP. The images were scanned at about 2 min after the addition of $500 \mathrm{nM} \mathrm{S} 1$. S1 molecules

bound on the top and along the side of the filaments are indicated by yellow and white arrowheads,

respectively. In (A), $500 \mathrm{nM} \mathrm{S1}$ was added to actin filaments. In (B), in contrast, actin filaments were incubated with $12.4 \mathrm{nM}$ Rng2CHD for $15 \mathrm{~min}$, then $500 \mathrm{nM} \mathrm{S1}$ was added. Bars: $50 \mathrm{~nm}$. (C) Number of S1 molecules bound on the top or along the sides of the filaments in the presence of $50 \mu \mathrm{M}$ ATP and $1 \mathrm{mM}$ ADP. The values were normalized by the total length of the measured filaments and time. 

made available under aCC-BY-NC 4.0 International license.

White bars: $500 \mathrm{nM} \mathrm{S1}$ was added to actin filaments. Red bars: Actin filaments were incubated with 12.4 nM Rng2CHD for $15 \mathrm{~min}$, then $500 \mathrm{nM} \mathrm{S1}$ was added. Blue bars: Actin filaments were incubated with $500 \mathrm{nM} \mathrm{S} 1$ in the nucleotide-free state for $10 \mathrm{~min}$, then $50 \mu \mathrm{M}$ ATP, $1 \mathrm{mM}$ ADP and $12.4 \mathrm{nM}$ Rng2CHD were added. The number of bound S1 molecules was counted in the images scanned between 1 and 2 min after the addition of S1 (white and red bars) or ATP (blue bars). 
Table 1 Estimated binding ratio of Rng2CHD and GFP-Rng2CHD to actin filaments.

$\begin{array}{ccccccc}\text { Inhibition rate of movement } & 50 \% & 75 \% & 80 \% & 90 \% & 95 \% & 100 \% \\ {\left[\mathrm{Rng}^{2} \mathrm{CHD}_{\text {bound }}\right] /\left[\text { Actin }_{\text {total }}\right]} & 1 / 75 & 1 / 30 & 1 / 28 & 1 / 21 & 1 / 13 & 1 / 6 \\ & & & & & & \\ {\left[\mathrm{GFP}-\mathrm{Rng}^{2} \mathrm{CHD}_{\text {bound }}\right] /\left[\text { Actin }_{\text {total }}\right]} & - & - & 1 / 10 & 1 / 5 & - & 1 / 2\end{array}$

The estimated binding ratio of Rng2CHD and GFP-Rng2CHD to actin protomers that caused various degrees of

movement inhibition. These values were estimated based on the following approximation: $\left[R n g 2 C H D_{\text {total }}\right] / K_{d} \cong$

$[$ Rng2CHD bound $] /\left[\right.$ Actin $\left._{\text {free }}\right]$ for Rng2CHD, and from fluorescence intensities for GFP-Rng2CHD (Fig. 3). 


\section{References}

1. I. Mabuchi, M. Okuno, The effect of myosin antibody on the division of starfish blastomeres. J. Cell Biol. 74, 251-263 (1977).

2. L. L. Satterwhite, T. D. Pollard, Cytokinesis. Cell Biol. 4, 43-52 (1992).

3. R. J. Pelham, F. Chang, Actin dynamics in the contractile ring during cytokinesis in fission yeast. Nature 419, 82-86 (2002).

4. M. Lord, T. D. Pollard, UCS protein Rng3p activates actin filament gliding by fission yeast myosin-II. J. Cell Biol. 167, 315-325 (2004).

5. T. Kamasaki, M. Osumi, I. Mabuchi, Three-dimensional arrangement of F-actin in the contractile ring of fission yeast. J. Cell Biol. 178, 765-771 (2007).

6. J. H. Zang, et al., On the role of myosin-II in cytokinesis: division of Dictyostelium cells under adhesive and nonadhesive conditions. Mol. Biol. Cell 8, 2617-2629 (1997).

7. S. J. Kron, J. A. Spudich, Fluorescent actin filaments move on myosin fixed to a glass surface. Proc. Natl. Acad. Sci. U. S. A. 83, 6272-6276 (1986).

8. A. V. Hill, The heat of shortening and the dynamic constants of muscle. Proc. R. Soc.

London. Ser. B - Biol. Sci. 126, 136-195 (1938).

9. K. Eng, N. I. Naqvi, K. C. Y. Wong, M. K. Balasubramanian, Rng2p, a protein required for cytokinesis in fission yeast, is a component of the actomyosin ring and the spindle 
pole body. Curr. Biol. 8, 611-621 (1998).

10. S. Mukhina, Y. Wang, M. Murata-Hori, Alpha-Actinin is Required for Tightly Regulated Remodeling of the Actin Cortical Network during Cytokinesis. Dev. Cell 13, 554-565

(2007).

11. Y. Li, et al., The F-actin bundler $\alpha$-actinin Ain 1 is tailored for ring assembly and constriction during cytokinesis in fission yeast. Mol. Biol. Cell 27, 1821-1833 (2016).

12. M. Takaine, O. Numata, K. Nakano, Fission yeast IQGAP arranges actin filaments into the cytokinetic contractile ring. EMBO J. 28, 3117-3131 (2009).

13. K. Tokuraku, R. Kurogi, R. Toya, T. Q. P. Uyeda, Novel Mode of Cooperative Binding between Myosin and $\mathrm{Mg}^{2+}$-actin Filaments in the Presence of Low Concentrations of ATP. J. Mol. Biol. 386, 149-162 (2009).

14. R. Hirakawa, Y. Nishikawa, T. Q. P. Uyeda, K. Tokuraku, Unidirectional growth of heavy meromyosin clusters along actin filaments revealed by real-time fluorescence microscopy.

Cytoskelet. 74, 482-489 (2017).

15. K. X. Ngo, et al., Allosteric regulation by cooperative conformational changes of actin filaments drives mutually exclusive binding with cofilin and myosin. Sci. Rep. 6, 1-11 (2016).

16. A. McGough, B. Pope, W. Chiu, A. Weeds, Cofilin changes the twist of F-actin: 
Implications for actin filament dynamics and cellular function. J. Cell Biol. 138, 771-781

(1997).

17. V. E. Galkin, A. Orlova, N. Lukoyanova, W. Wriggersd, E. H. Egelman, Actin

depolymerizing factor stabilizes an existing state of F-actin and can change the tilt of F-

actin subunits. J. Cell Biol. 153, 75-86 (2001).

18. K. X. Ngo, N. Kodera, E. Katayama, T. Ando, T. Q. P. Uyeda, Cofilin-induced

unidirectional cooperative conformational changes in actin filaments revealed by high-

speed atomic force microscopy. Elife 4, 1-19 (2015).

19. A. Orlova, E. Prochniewicz, E. H. Egelman, Structural Dynamics of F-Actin: II.

Cooperativity in Structural Transitions. J. Mol. Biol. 245, 598-607 (1995).

20. F. Oosawa, S. Fujime, S. Ishiwata, K. Mihashi, Dynamic Property of F-Actin and Thin

Filament. Cold Spring Harb. Symp. Quant. Biol. 37, 277-285 (1973).

21. S. Khaitlina, O. Tsaplina, H. Hinssen, Cooperative effects of tropomyosin on the dynamics of the actin filament. FEBS Lett. 591, 1884-1891 (2017).

22. M. C. Craig-Schmidt, R. M. Robson, D. E. Goll, M. H. Stromer, Effect of alpha-actinin on actin structure. Biochim. Biophys. Acta - Protein Struct. 670, 9-16 (1981).

23. G. Papp, et al., Conformational changes in actin filaments induced by formin binding to the barbed end. Biophys. J. 91, 2564-2572 (2006). 
24. H. Huxley, The Mechanism of Muscular Contraction. Science 164, 1356-1366 (1969).

25. R. Cooke, M. S. Crowder, C. H. Wendt, V. A. Barnett, D. D. Thomas, Muscle Cross-

Bridges: Do They Rotate? Adv. Exp. Med. Biol. 170, 413-427 (1984).

26. M. Tokunaga, K. Sutoh, T. Wakabayashi, Structure and Structural Change of the Myosin

Head. Adv. Biophys. 27, 157-167 (1991).

27. T. Q. P. Uyeda, P. D. Abramson, J. A. Spudich, The neck region of the myosin motor domain acts as a lever arm to generate movement. Proc. Natl. Acad. Sci. 93, 4459-4464 (1996).

28. R. W. Lymn, E. W. Taylor, Mechanism of Adenosine Triphosphate Hydrolysis by Actomyosin. Biochemistry 10, 4617-4624 (1971).

29. J. N. Forkey, M. E. Quinlan, M. A. Shaw, J. E. T. Corrie, Y. E. Goldman, Threedimensional structural dynamics of myosin V by single-molecule fluorescence polarization. Nature 422, 399-404 (2003).

30. N. Kodera, D. Yamamoto, R. Ishikawa, T. Ando, Video imaging of walking myosin v by high-speed atomic force microscopy. Nature 468, 72-76 (2010).

31. H. Kubota, S. V. Mikhailenko, H. Okabe, H. Taguchi, S. Ishiwata, D-loop of actin differently regulates the motor function of myosins II and V. J. Biol. Chem. 284, 3525135258 (2009). 
32. T. Q. P. Noguchi, et al., G146V mutation at the hinge region of actin reveals a myosin

class-specific requirement of actin conformations for motility. J. Biol. Chem. 287, 24339-

24345 (2012).

33. K. Iwase, M. Tanaka, K. Hirose, T. Q. P. Uyeda, H. Honda, Acceleration of the sliding

movement of actin filaments with the use of a non-motile mutant myosin in in vitro

motility assays driven by skeletal muscle heavy meromyosin. PLoS One 12, 1-13 (2017).

34. T. Q. P. Uyeda, K. Tokuraku, K. Kaseda, M. R. Webb, B. Patterson, Evidence for a

Novel, Strongly Bound Acto - S1 Complex Carrying ADP and Phosphate Stabilized in the

G680V Mutant of Dictyostelium Myosin II. Biochemistry 41, 9525-9534 (2002).

35. M. Preller, et al., Structural basis for the allosteric interference of myosin function by

reactive thiol region mutations G680A and G680V. J. Biol. Chem. 286, 35051-35060

(2011).

36. S. Fujime, Phosphorylation of Myosin Light Chain Modulates the In Vitro Movement of

Fibers Composed of Actin and Myosin from Skeletal Muscle. J. Biochem. 94, 1539-1545

(1983).

37. J. R. Sellers, J. A. Spudich, M. P. Sheetz, Light chain phosphorylation regulates the movement of smooth muscle myosin on actin filaments. J. Cell Biol. 101, 1897-1902 (1985). 
38. L. M. Griffith, S. M. Downs, J. A. Spudich, Myosin light chain kinase and myosin light chain phosphatase from Dictyostelium: Effects of reversible phosphorylation on myosin structure and function. J. Cell Biol. 104, 1309-1323 (1987).

39. S. Ebashi, A. Kodama, A new protein factor promoting aggregation of tropomyosin. $J$. Biochem. 58, 107-108 (1965).

40. S. Ebashi, A. Kodama, Interacton of Troponin with F-actin in the Presence of Tropomyosin. J. Biochem. 59, 425-426 (1966).

41. V. P. Shirinsky, K. G. Biryukov, J. M. Hettasch, J. R. Sellers, Inhibition of the relative movement of actin and myosin by caldesmon and calponin. J. Biol. Chem. 267, 15886$15892(1992)$.

42. J. M. Chalovich, P. Cornelius, C. E. Benson, Caldesmon inhibits skeletal actomyosin subfragment-1 ATPase activity and the binding of myosin subfragment-1 to actin. J. Biol.

Chem. 262, 5711-5716 (1987).

43. J. Kolakowski, A. Karkucinska, R. Dabrowska, Calponin inhibits actin-activated MgATPase of myosin subfragment 1 (S1) without displacing S1 from its binding site on actin. Eur. J. Biochem. 243, 624-629 (1997).

44. I. R. Tebbs, T. D. Pollard, Separate roles of IQGAP Rng2p in forming and constricting the Schizosaccharomyces pombe cytokinetic contractile ring. Mol. Biol. Cell 24, 1904- 
1917 (2013).

45. J. A. Spudich, S. Watt, The Regulation of Rabbit Skeletal Muscle Contraction. J. Biol.

Chem 246, 4866-4871 (1971).

46. J. D. Pardee, J. A. Spudich, Purification of muscle actin. Methods Enzymol. 85, 164-181

(1982).

47. S. S. Margossian, S. Lowey, Preparation of myosin and its subfragments. Meth.Enzymol.

85, 55-71 (1982).

48. S. Watanabe, R. Ikebe, M. Ikebe, Drosophila myosin VIIA is a high duty ratio motor with a unique kinetic mechanism. J. Biol. Chem. 281, 7151-7160 (2006).

49. C. A. Schneider, W. S. Rasband, K. W. Eliceiri, NIH Image to ImageJ: 25 years of Image Analysis HHS Public Access. Nat. Methods 9, 671-675 (2012).

50. E. Meijering, O. Dzyubachyk, I. Smal, Methods for Cell and Particle Tracking. Methods Enzymol. 504, 183-200 (2012).

51. H. Shishido, M. D. Yamada, K. Kondo, S. Maruta, Photocontrol of calmodulin interaction with target peptides using azobenzene derivative. J. Biochem. 146, 581-590 (2009).

52. T. Kodama, K. Fukui, K. Kometani, The initial phosphate burst in ATP hydrolysis by myosin and subfragment- 1 as studied by a modified malachite green method for determination of inorganic phosphate. J. Biochem. 99, 1465-1472 (1986). 
bioRxiv preprint doi: https://doi.org/10.1101/2020.04.14.041046; this version posted May 23, 2020. The copyright holder for this preprint (which was not certified by peer review) is the author/funder, who has granted bioRxiv a license to display the preprint in perpetuity. It is made available under aCC-BY-NC 4.0 International license.

53. T. Ando, T. Uchihashi, N. Kodera, High-Speed AFM and Applications to Biomolecular

Systems. Annu. Rev. Biophys. 42, 393-414 (2013). 


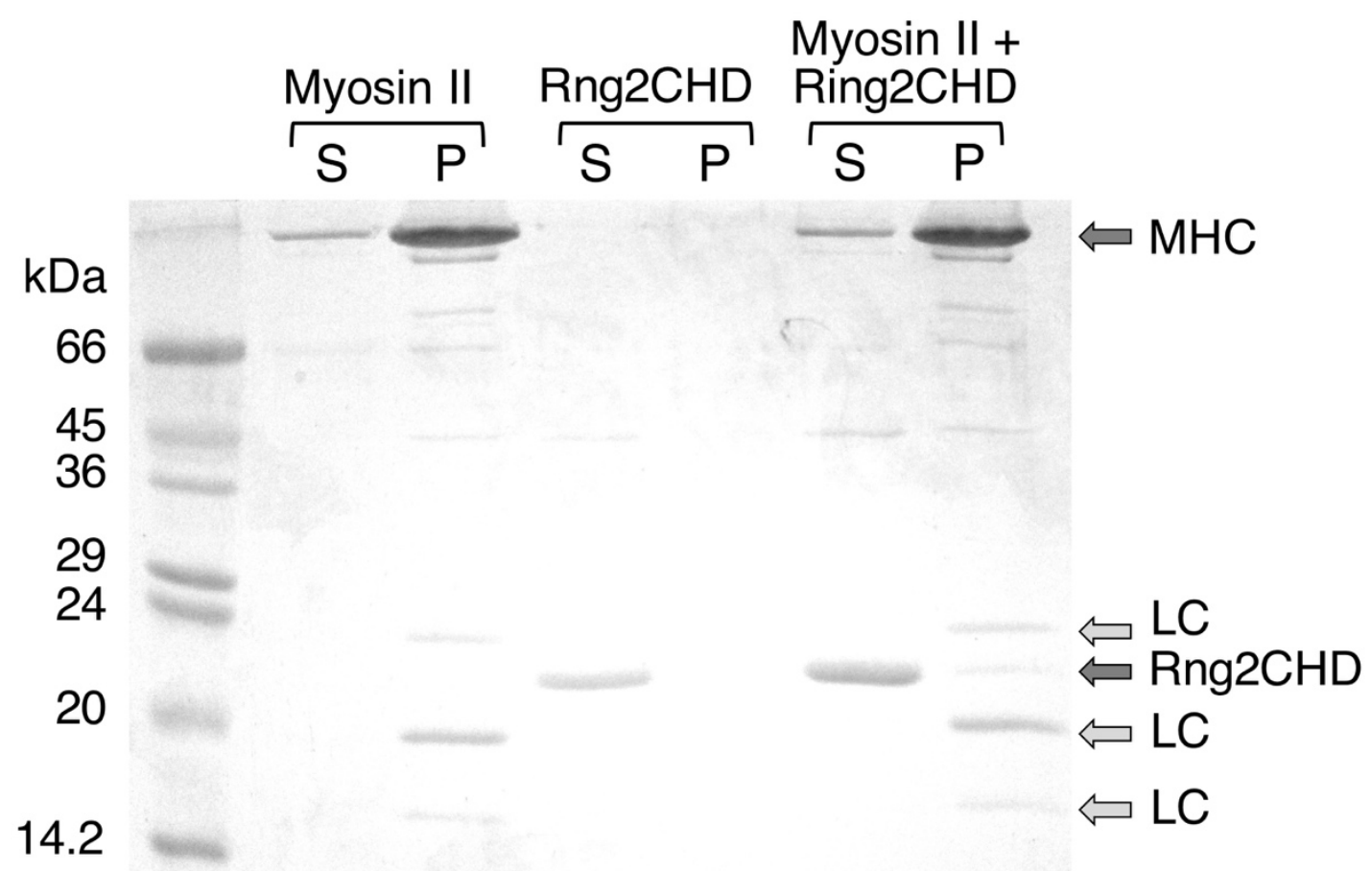

Supplemental Figure 1 Co-sedimentation of Rng2CHD with skeletal muscle myosin II

\section{filaments.}

Skeletal muscle myosin II filaments, Rng2CHD and a mixture of the two proteins were centrifuged at $30,000 \mathrm{rpm}$ for $10 \mathrm{~min}$ at $22^{\circ} \mathrm{C}$ in a Beckman TLA100.1 rotor, and the supernatant and pellet fractions were analyzed by SDS-PAGE. The concentrations of myosin II and Rng2CHD were $2 \mu \mathrm{M}$, and the buffer was $10 \mathrm{mM}$ Hepes (pH 7.4), $25 \mathrm{mM} \mathrm{KCl}, 4 \mathrm{mM} \mathrm{MgCl} 2,0.2 \mathrm{mM}$ ATP and $1 \mathrm{mM}$ DTT. 